\title{
Expectations of the reductions for type-2 trapezoidal fuzzy variables and its application to a multi-objective solid transportation problem via goal programming technique
}

Anushree Dutta ${ }^{1}$ and Dipak Kumar Jana ${ }^{2^{*}}$ (D)

\author{
*Correspondence: \\ dipakjana@gmail.com \\ ${ }^{2}$ Department of Applied Science, \\ Haldia Institute of Technology, \\ Haldia, Purba Midnapur, Pin-721657, \\ West Bengal, India \\ Full list of author information is \\ available at the end of the article
}

\begin{abstract}
In this paper, based on the concepts of credibility measure and expectation theory, we derive the expectation formulae for the three reductions of a type-2 trapezoidal fuzzy variable (T2TrFV), which are attained by adopting the critical value (CV) reduction methods. We minimize the total transportation cost and the total transportation time over a single layered distribution system consisting of vendors and customers represented as a multi-objective solid transportationproblem. To portray the uncertainty in a real life choice environment, we consider the unit cost of transportation, demands, availabilities, conveyance capacities, unit transportation time and unit loading and unloading time as T2TrFVs. The corresponding deterministic model, which is obtained by the application of expectation formulas deduced earlier, is converted to a single objective optimization problem using goal programming technique and weighted sum method via the soft computing technique-generalized reduced gradient (LINGO-14.0). A numerical experiment is finally illustrated and corresponding graphical representations are provided.
\end{abstract}

Keywords: Fuzzy type-2 trapezoidal variables, CV reduction methods, Expected value, Solid transportation problem, Goal programming technique, Weighted sum method

\section{Introduction}

The thought of type-2 fuzzy set (T2FS) was first introduced by Zadeh [1] as an extension of the concept of an ordinary fuzzy set or a type-1 fuzzy set (T1FS). Much work has been done from then on based on type-2 fuzzy set theory. For examples, Karnik et al. [2] introduced a type-2 fuzzy logic system, which takes care of rule uncertainties; Liang and Mendel [3] presented the theory and design of interval type-2 fuzzy logic systems; Karnik and Mendel [4] discussed set operations on T2FSs; Chen and Chang [5] studied fuzzy rule interpolation based on the ratio of fuzziness of interval type- 2 fuzzy sets; Abdullah and Najib [6] used linguistic variables in interval type-2 fuzzy sets (IT2FSs) and a rank value method to normalize upper and lower memberships of IT2FS.

Zadeh [7] gave the definition of type-1 fuzzy sets (T1FSs), and later in Zadeh [1] elaborated on the fact that in fuzzy logic everything is allowed to be a matter of degree (where the degree could be fuzzy) which led to the introduction of T2FS. But it is quite difficult

(c) The Author(s), 2017 Open Access This article is distributed under the terms of the Creative Commons Attribution 4.0 International License (http://creativecommons.org/licenses/by/4.0/), which permits unrestricted use, distribution, and reproduction in any medium, provided you give appropriate credit to the original author(s) and the source, provide a link to the Creative Commons license, and indicate if changes were made. 
to deal with a type-2 fuzzy variable (T2FV) more than a type-1 fuzzy variable (T1FV) since T2FV has the possibility of taking a crisp value and the possibility is again a fuzzy number in $[0,1]$, i.e, due to the fuzzy membership function of a type-2 fuzzy number, the computation complexity is very high in practical applications. To overcome this difficulty, few reduction methods have been used in the literature, where T2FV are reduced to T1FV and then defuzzification of T1FV is carried out. Karnik and Mendel [8] introduced a method for type reduction via the concept of a centroid of a T2FS; Coupland [9] presented the geometric defuzzification for generalized T2FSs; Liu [10] employed a centroid type reduction strategy for a general type-2 fuzzy logic system; to defuzzify T2 fuzzy variable, Qin et al. [11] presented three types of CV reduction methods for the T2 fuzzy variable; Chen [12] deduced the generalized expectation for three reductions of type-2 triangular fuzzy variable (T2TFV). In this paper, we deduce the expectation formulas of the reductions for type- 2 trapezoidal fuzzy variable using $\mathrm{CV}$ reduction methods, which will be useful in solving some optimization problems with T2 fuzzy coefficients.

The solid transportation problem (STP) was first proposed by Haley [13]. The STP is an extension of the classical transportation problem (TP), as the addition of multiple conveyances. Since the introduction of STP, researchers have been using different solution procedures to solve STPs, when formulated under fuzzy environment. Chanas et al. [14] formulated and solved TPs under fuzzy environment. Some recent works in this context are Sakawa [15], who applied fuzzy goal programming method for solving multi-objective nonlinear programming problem; Ojha et al. [16] presented a STP for item with fixed charge, vehicle cost and price discounted varying charge using genetic algorithm; Fegad et al. [17] used interval and triangular membership functions in a TP. A few recent works in the field of TP and STP are that of Zavardehi et al. [18], Figueroa-Garc and Hernandez [19], Kaur and Kumar [20], Jana et al. [21], Tao and Xu [22], Kundu et al. [23], Sadeghi et al. [24], Liu et al. [25] etc. Yang et al. [26] applied methods of reduction for type-2 fuzzy variables to STP. Jana et al. [27] presented application of CV and mean reduction methods on T2FS to a multilevel profit TP. In this paper, we have for the first time, solved a STP under type-2 trapezoidal fuzzy (T2TrF) environment using CV reduction method and expectation formulas of the reductions via Goal Programming Technique (GPT).

During transportation activities, some important parameters of a STP, such as demands at the destinations, the supply capacities at the origins, the capacities of the conveyances, the time of transportation, loading and unloading time at the origins and destinations respectively, etc., can be considered to be fuzzy variables, depending on real life requiments. In such situations, it becomes more reasonable to use fuzzy optimization methods to find the solution of the STP. As a result, to fit the demands of an uncertain environment, we introduce type- 2 trapezoidal fuzzy variable in STP.

In this paper, we have two motivations. First, to deduce the expectation formulas of the reductions of a $\mathrm{T} 2 \mathrm{TrFV}$, employing the $\mathrm{CV}$ reduction method, which can later be utilized in solving problems involving trapezoidal type reduction. Second, to study the STP problem involving type-2 trapezoidal fuzzy variables, use of which is quite practical keeping real life uncertain situations in mind. Working with type-2 fuzzy variables is more difficult than type- 1 fuzzy variables and hence this is where the type reduction method becomes helpful. With these ideas in mind, we are interested in how to draw up the solid transportation model, and then design effective algorithms to produce the optimal transportation strategies. To this end, this study proposes a defuzzification method for type-2 
fuzzy variables via general expectation method. Numerical experiments are carried out using soft computing technique Lingo-14.0.

The major objectives of the proposed research work are as follows:

- Expectations of the reductions for type-2 trapezoidal fuzzy variables have been deduced.

- A new class of generalized expectation method has been introduced for type-2 trapezoidal fuzzy variables.

- Multi-objective optimization problems have been converted into a single objective optimization problem using goal programming technique and weighted sum method.

- Comparative results for type-2 and type-1 trapezoidal fuzzy variables are reported with some different optimistic levels and different weights of objective functions.

The structure of this paper is as follows. In Section "Preliminary ideas on type-2 fuzzy sets", we give some preliminaries about T2FS. In Section "Expected value for reduction of a T2 trapezoidal fuzzy variable", we intent to deduce the expectation formulas of three reductions of T2 trapezoidal fuzzy variable. In Section "Fuzzy goal programming method", we present the concept of Goal Programming Technique. In Section "Notations", notations of the proposed model are presented. In Section "Formulation of type 2 fuzzy solid transportation problem (T2FSTP)", we formulate the model in fuzzy T2 trapezoidal environment. In Section "Equivalent crisp problem in T2", we present the equivalent crisp model. A numerical experiment and solution procedure via goal programming technique is presented in Section "Numerical experiment". Experimental results and discussion are presented in Section "Discussion". The paper is concluded in Section "Conclusions".

\section{Preliminary ideas on type-2 fuzzy sets}

The idea of type-2 fuzzy sets was first introduced by Prof. Zadeh in 1975 [1], as an extension of ordinary fuzzy sets, whose truth values are ordinary fuzzy sets in the unit interval, i.e., fuzzy truth values. The overviews on type-2 fuzzy sets were given in [28]. Since ordinary fuzzy sets and interval-valued fuzzy sets are special cases of type- 2 fuzzy sets, Takc [29] described that type-2 fuzzy sets are very useful in circumstances where there is need to handle more uncertainties than it is possible using ordinary fuzzy sets or intervalvalued fuzzy sets. Recently, Karnik et al. [2] proposed type-2 fuzzy logic systems from the view of type reduction and centroid method. A type-2 fuzzy set (Jana et al. [30]) expresses the non-deterministic truth degree with imprecision and uncertainty for an element that belongs to a set.

A type-2 fuzzy set denoted by $\tilde{\tilde{A}}$, is characterized by a type- 2 membership function $\mu_{\tilde{A}}(x, u)$ where $x \in X, \forall u \in J_{x}^{u} \subseteq[0,1]$ and $0 \leq \mu_{\tilde{A}}(x, u) \leq 1$ defined in Eq. (1).

$$
\tilde{\tilde{A}}=\left\{\left(x, u, \mu_{\tilde{A}}(x, u)\right) \mid x \in X, \forall u \in J_{x}^{u} \subseteq[0,1]\right\}
$$

If $\tilde{\tilde{A}}$ is fuzzy type-2 (FT2) continuous variable, it is denoted in Eq. (2)

$$
\tilde{\tilde{A}}=\left\{\int_{x \in X}\left[\int_{u \in J_{x}^{u}} f_{x}(u) / u\right] / x\right\}
$$


where $\iint$ denotes the union of $x$ and $u$. If $\tilde{\tilde{A}}$ is FT2 discrete, then it is denoted by Eq. (3)

$$
\tilde{\tilde{A}}=\left\{\sum_{x \in X} \mu_{\tilde{\tilde{A}}}(x) / x\right\}=\left\{\sum_{i=1}^{N}\left[\sum_{k=1}^{M_{i}} f_{x_{i}}\left(u_{k}\right) / u_{i k}\right] / x_{i}\right\}
$$

where $\sum \sum$ denotes the union of $x$ and $u$.

If $f_{x}(u)=1, \forall u \in\left[J_{-}^{u}, J_{x}^{u}\right] \subseteq[0,1]$, the type- 2 membership function $\mu_{\tilde{A}}(x, u)$ is expressed by one type- 1 inferior membership function, $J_{-x}^{u}=\mu_{A}(x)$ and one type- 1 superior, $\bar{J}_{x}^{u}=$ $\mu_{A}(x)$, then it is called an interval type-2 fuzzy set, denoted by Eqs. (4) and (5).

$$
\tilde{\tilde{A}}=\left\{(x, u, 1) \mid \forall x \in X, \forall u \in\left[\underline{\mu}_{A}(x), \bar{\mu}_{A}(x)\right] \subseteq[0,1]\right\}
$$

or

$$
\begin{aligned}
\tilde{\tilde{A}} & =\left\{\int_{x \in X}\left[\int_{u \in\left[\left[_{-x}^{u} \bar{J}_{x}^{u}\right] \subseteq[0,1]\right.} 1 / u\right] / x\right\} \\
& =\left\{\int_{x \in X}\left[\int_{u \in\left[\underline{\mu}_{A}(x), \bar{\mu}_{A}(x)\right] \subseteq[0,1]} 1 / u\right] / x\right\}
\end{aligned}
$$

Definition 1 [25] A type-2 trapezoidal fuzzy variable $\tilde{\xi}$ is denoted by $\tilde{\xi}=$ $\left(r_{1}, r_{2}, r_{3}, r_{4}, \theta_{r}, \theta_{l}\right)$, where $r_{1}, r_{2}, r_{3}, r_{4}$ are real values and $\theta_{r}, \theta_{l} \in[0,1]$ are two parameters characterizing the degree of uncertainty that $\tilde{\tilde{\xi}}$ takes a value $x$.

For $x \in\left[r_{1}, r_{2}\right]$, the secondary possibility distribution function $\tilde{\mu}_{\tilde{\xi}}(x)$ of $\tilde{\tilde{\xi}}$ is defined in the form

$$
\tilde{\mu}_{\tilde{\xi}}(x)=\left(\frac{x-r_{1}}{r_{2}-r_{1}}+\theta_{l} \min \left\{\frac{x-r_{1}}{r_{2}-r_{1}}, \frac{r_{2}-x}{r_{2}-r_{1}}\right\}, \frac{x-r_{1}}{r_{2}-r_{1}}, \frac{x-r_{1}}{r_{2}-r_{1}}+\theta_{r} \min \left\{\frac{x-r_{1}}{r_{2}-r_{1}}, \frac{r_{2}-x}{r_{2}-r_{1}}\right\}\right)
$$

For $x \in\left(r_{2}, r_{3}\right]$, the secondary possibility distribution function $\tilde{\mu}_{\tilde{\xi}}(x)$ of $\tilde{\tilde{\xi}}$ is 1 .

For $x \in\left(r_{3}, r_{4}\right]$, the secondary possibility distribution function $\tilde{\mu}_{\tilde{\xi}}$ of $\tilde{\tilde{\xi}}$ is defined in the form

$\tilde{\mu}_{\tilde{\xi}}=\left(\frac{r_{4}-x}{r_{4}-r_{3}}-\theta_{l} \min \left\{\frac{r_{4}-x}{r_{4}-r_{3}}, \frac{x-r_{3}}{r_{4}-r_{3}}\right\}, \frac{r_{4}-x}{r_{4}-r_{3}}, \frac{r_{4}-x}{r_{4}-r_{3}}+\theta_{r} \min \left\{\frac{r_{4}-x}{r_{4}-r_{3}}, \frac{x-r_{3}}{r_{4}-r_{3}}\right\}\right)$

Definition 2 [31] Let $\Gamma$ be the universe of discourse. An ample field $\mathcal{A}$ on $\Gamma$ is a class of subsets of $\Gamma$ that is closed under arbitrary unions, intersections, and complements in $\Gamma$.

Let Pos : $A \longrightarrow[0,1]$ be a set function on the ample field $\mathcal{A}$. Pos is said to be a possibility measure if it satisfies the following conditions:

$P 1: \operatorname{Pos}(\Phi)=0$ and $\operatorname{Pos}(\Gamma)=1$.

P2: For any subclass $\left\{A_{i} \mid i \in I\right\}$ of $\mathcal{A}$ (finite, countable or uncountable),

$$
\operatorname{Pos}\left(\bigcup_{i=I} A_{i}\right)=\sup _{i=I} \operatorname{Pos}\left(A_{i}\right)
$$


The triplet $(\Gamma, \mathcal{A}$, Pos) is referred to as a possibility space, in which a credibility measure is defined as

$$
\operatorname{Cr}(A)=\frac{1}{2}\left(1+\operatorname{Pos}(A)-\operatorname{Pos}\left(A^{c}\right)\right), A \in \mathcal{A}
$$

Definition 3 [11] Let $\tilde{\xi}$ be an RFV. Then the optimistic CV of $\tilde{\xi}$, denoted by $C V^{*}[\tilde{\xi}]$ is defined as

$$
C V^{*}[\tilde{\xi}]=\sup _{\alpha \in[0,1]}[\alpha \wedge \operatorname{Pos}(\tilde{\xi} \geq \alpha)],
$$

while the pessimistic $C V$ of $\tilde{\xi}$, denoted by $C V_{*}[\tilde{\xi}]$, is defined as

$$
C V_{*}[\tilde{\xi}]=\sup _{\alpha \in[0,1]}[\alpha \wedge \operatorname{Nec}(\tilde{\xi} \geq \alpha)],
$$

The $C V$ of $\tilde{\xi}$, denoted by $C V[\tilde{\xi}]$, is defined

$$
C V[\tilde{\xi}]=\sup _{\alpha \in[0,1]}[\alpha \wedge \operatorname{Cr}(\tilde{\xi} \geq \alpha)],
$$

The formulas for CVs of a triangular $R F V \tilde{\xi}=\left(r_{1}, r_{2}, r_{3}\right)$ are:

(i) The optimistic $C V$ of $\tilde{\xi}$ is:

$$
C V^{*}[\tilde{\xi}]=\frac{r_{3}}{1+r_{3}-r_{2}}
$$

(ii) The pessimistic CV of $\tilde{\xi}$ is:

$$
C V_{*}[\tilde{\xi}]=\frac{r_{2}}{1+r_{2}-r_{1}}
$$

(iii) The CV of $\tilde{\xi}$ is:

$$
C V[\tilde{\xi}]= \begin{cases}\frac{2 r_{2}-r_{1}}{1+2\left(r_{2}-r_{1}\right)}, \text { if } \quad r_{2}>\frac{1}{2} \\ \frac{r_{3}}{1+2\left(r_{3}-r_{2}\right)}, \text { if } \quad r_{2} \leq \frac{1}{2}\end{cases}
$$

\section{Expected value for reduction of a T2 trapezoidal fuzzy variable}

Theorem 1 Assume $\tilde{\xi}_{1}$ to be the reduction of the type-2 trapezoidal fuzzy variable $\tilde{\tilde{\xi}}=$ $\left(r_{1}, r_{2}, r_{3}, r_{4}, \theta_{l}, \theta_{r}\right)$, obtained by the optimistic critical value reduction method. Then

$$
E\left[\tilde{\xi}_{1}\right]=\frac{r_{1}+r_{4}}{2}-\frac{\left(r_{1}-r_{2}-r_{3}+r_{4}\right) \ln \left(1+\frac{\theta_{r}}{2}\right)}{2 \theta_{r}}
$$

Proof For $x \in\left[r_{1}, r_{2}\right]$, the secondary possibility distribution function $\tilde{\mu}_{\tilde{\xi}}(x)$ of $\tilde{\tilde{\xi}}$ is the triangular RFV

$\tilde{\mu}_{\tilde{\xi}}(x)=\left(\frac{x-r_{1}}{r_{2}-r_{1}}+\theta_{l} \min \left\{\frac{x-r_{1}}{r_{2}-r_{1}}, \frac{r_{2}-x}{r_{2}-r_{1}}\right\}, \frac{x-r_{1}}{r_{2}-r_{1}}, \frac{x-r_{1}}{r_{2}-r_{1}}+\theta_{r} \min \left\{\frac{x-r_{1}}{r_{2}-r_{1}}, \frac{r_{2}-x}{r_{2}-r_{1}}\right\}\right)$

For $x \in\left(r_{2}, r_{3}\right]$, the secondary possibility distribution function $\tilde{\mu}_{\tilde{\xi}}(x)$ of $\tilde{\tilde{\xi}}$ is 1

For $x \in\left(r_{3}, r_{4}\right]$, the secondary possibility distribution function $\tilde{\mu}_{\tilde{\xi}}$ of $\tilde{\xi}$ is the triangular RFV

$\tilde{\mu}_{\tilde{\xi}}=\left(\frac{r_{4}-x}{r_{4}-r_{3}}-\theta_{l} \min \left\{\frac{r_{4}-x}{r_{4}-r_{3}}, \frac{x-r_{3}}{r_{4}-r_{3}}\right\}, \frac{r_{4}-x}{r_{4}-r_{3}}, \frac{r_{4}-x}{r_{4}-r_{3}}+\theta_{r} \min \left\{\frac{r_{4}-x}{r_{4}-r_{3}}, \frac{x-r_{3}}{r_{4}-r_{3}}\right\}\right)$ 
Then, according to Eq. (14) , for a trapezoidal fuzzy variable, the possibility distribution function $\tilde{\xi}_{1}$ is given by

$$
\begin{aligned}
& \mu_{\tilde{\xi}_{1}}(x)= \operatorname{Pos}\left\{\tilde{\xi}_{1}=x\right\} \\
& \Rightarrow \mu_{\tilde{\xi}_{1}}(x)= \begin{cases}\frac{\frac{\left(x-r_{1}\right)}{r_{2}-r_{1}}+\theta_{r} \min \left\{\frac{x-r_{1}}{r_{2}-r_{1}}, \frac{r_{2}-x}{r_{2}-r_{1}}\right\}}{1+\theta_{r} \min \left\{\frac{x-r_{1}}{r_{2}-r_{1}}, \frac{r_{2}-x}{r_{2}-r_{1}}\right\}}, & \text { if } x \in\left[r_{1}, r_{2}\right] \\
1, & \text { if } x \in\left(r_{2}, r_{3}\right] \\
\frac{r_{4}-x}{r_{4}-r_{3}}+\theta_{r} \min \left\{\frac{r_{4}-x}{r_{4}-r_{3}}, \frac{x-r_{3}}{\left.r_{4}-r_{3}\right\}}\right. & \text { if } x \in\left(r_{3}, r_{4}\right]\end{cases} \\
& \Rightarrow \mu_{\tilde{\xi}_{1}}(x)=\left\{\begin{array}{lll}
\frac{\left(1+\theta_{r} \min \left\{\frac{r_{4}-x}{r_{4}-r_{3}}, \frac{x-r_{3}}{r_{4}-r_{3}}\right\}\right.}{\left(r_{2}-r_{1}\right)+\theta_{r}\left(x-r_{1}\right)}, & \text { if } & x \in\left[r_{1}, \frac{r_{1}+r_{2}}{2}\right] \\
\frac{\left(1-\theta_{r}\right) x+\theta_{r} r_{2}-r_{1}}{\left(r_{2}-r_{1}\right)+\theta_{r}\left(r_{2}-x\right)}, & \text { if } & x \in\left(\frac{r_{1}+r_{2}}{2}, r_{2}\right] \\
1, & \text { if } & x \in\left(r_{2}, r_{3}\right] \\
\frac{\left(\theta_{r}-1\right) x-\theta_{r} r_{3}+r_{4}}{\left(r_{4}-r_{3}\right)+\theta_{r}\left(x-r_{3}\right)}, & \text { if } & x \in\left(r_{3}, \frac{r_{3}+r_{4}}{2}\right] \\
\frac{\left(1+\theta_{r}\right)\left(r_{4}-x\right)}{\left(r_{4}-r_{3}\right)+\theta_{r}\left(r_{4}-x\right)}, & \text { if } & x \in\left(\frac{r_{3}+r_{4}}{2}, r_{4}\right]
\end{array}\right.
\end{aligned}
$$

Therefore the credibility of $\tilde{\xi}_{1}$, using Eq. (10), is defined by

$$
\operatorname{Cr}\left\{\tilde{\xi}_{1} \geq r\right\}=\left\{\begin{array}{lll}
1, & \text { if } & r<r_{1} \\
1-\frac{\left(1+\theta_{r}\right)\left(r-r_{1}\right)}{2\left(r_{2}-r_{1}+\theta_{r}\left(r-r_{1}\right)\right)}, & \text { if } & r \in\left[r_{1}, \frac{r_{1}+r_{2}}{2}\right] \\
1-\frac{\left(1-\theta_{r} r+r+r_{r} r_{2}-r_{1}\right)}{2\left(r_{2}-r_{1}+\theta_{r}\left(r-r_{1}\right)\right)}, & \text { if } & r \in\left(\frac{r_{1}+r_{2}}{2}, r_{2}\right] \\
\frac{1}{2}, & \text { if } & r \in\left(r_{2}, r_{3}\right] \\
\frac{\left(\theta_{r}-1\right) r-\theta_{r} r_{3}+r_{4}}{2\left(r_{4}-r_{3}+\theta_{r}\left(r-r_{3}\right)\right)}, & \text { if } & r \in\left(r_{3}, \frac{r_{3}+r_{4}}{2}\right] \\
\frac{\left(1+\theta_{r}\left(r_{4}-r\right)\right.}{2\left(r_{4}-r_{3}+\theta_{r}\left(r_{4}-r\right)\right)}, & \text { if } & r \in\left(\frac{r_{3}+r_{4}}{2}, r_{4}\right] \\
0, & \text { if } & r>r_{4}
\end{array}\right.
$$

and

$$
\operatorname{Cr}\left\{\tilde{\xi}_{1} \leq r\right\}=\left\{\begin{array}{lll}
0, & \text { if } & r<r_{1} \\
\frac{\left(1+\theta_{r}\right)\left(r-r_{1}\right)}{2\left(r_{2}-r_{1}+\theta_{r}\left(r-r_{1}\right)\right)}, & \text { if } & r \in\left[r_{1}, \frac{r_{1}+r_{2}}{2}\right] \\
\frac{\left.\left(1-\theta_{r}\right) r+\theta_{r} r_{2}-r_{1}\right)}{2\left(r_{2}-r_{1}+\theta_{r}\left(r_{2}-r\right)\right)}, & \text { if } & r \in\left(\frac{r_{1}+r_{2}}{2}, r_{2}\right] \\
\frac{1}{2}, & \text { if } & r \in\left(r_{2}, r_{3}\right] \\
1-\frac{\left(\theta_{r}-1\right) r-\theta_{r} r_{3}+r_{4}}{2\left(r_{4}-r_{3}+r_{r}\left(r-r_{3}\right)\right)}, & \text { if } & r \in\left(r_{3}, \frac{r_{3}+r_{4}}{2}\right] \\
1-\frac{\left(1+r_{r}\right)\left(r_{4}-r\right)}{2\left(r_{4}-r_{3}+\theta_{r}\left(r_{4}-r\right)\right)}, & \text { if } & r \in\left(\frac{r_{3}+r_{4}}{2}, r_{4}\right] \\
1, & \text { if } & r>r_{4}
\end{array}\right.
$$


If $r_{1} \geq 0$, then we have

$$
\left.\begin{array}{rl}
E\left[\tilde{\xi}_{1}\right]= & \int_{0}^{\infty} \operatorname{Cr}\left\{\tilde{\xi}_{1} \geq r\right\} d r-\int_{-\infty}^{0} \operatorname{Cr}\left\{\tilde{\xi}_{1} \leq r\right\} d r \\
= & \int_{0}^{r_{1}} 1 d r+\int_{r_{1}}^{\frac{r_{1}+r_{2}}{2}} 1 d r-\int_{r_{1}}^{\frac{r_{1}+r_{2}}{2}} \frac{\left(1+\theta_{r}\right)\left(r-r_{1}\right)}{\left(r_{2}-r_{1}\right)+\theta_{r}\left(r-r_{1}\right)} d r+\int_{\frac{r_{1}+r_{2}}{2}}^{r_{2}} 1 d r \\
& -\int_{\frac{r_{1}+r_{2}}{2}}^{r_{2}} \frac{\left(1-\theta_{r}\right) r+\theta_{r} r_{2}-r_{1}}{r_{2}-r_{1}+\theta_{r}\left(r_{2}-r\right)} d r+\int_{r_{2}}^{r_{3}} \frac{1}{2} d r+\int_{r_{3}}^{\frac{r_{3}+r_{4}}{2}} \frac{\left(\theta_{r}-1\right) r-\theta_{r} r_{3}+r_{4}}{2\left(r_{4}-r_{3}+\theta_{r}\left(r-r_{4}\right)\right.} d r \\
& +\int_{\frac{r_{3}+r_{4}}{2}}^{r_{4}} \frac{\left(1+\theta_{r}\right)\left(r_{4}-r\right)}{2\left(r_{4}-r_{3}+\theta_{r}\left(r_{4}-r\right)\right)} d r-0 \\
= & r_{1}+\frac{r_{1}+r_{2}}{2}-r_{1}+r_{2}-\frac{r_{1}+r_{2}}{2}+\frac{r_{3}-r_{2}}{2}-\frac{1}{2}\left[\frac{\left(1+\theta_{r}\right)\left(r_{2}-r_{1}\right)}{2 \theta_{r}}\right. \\
& +\frac{\left(1+\theta_{r}\right)\left(r_{2}-r_{1}\right) \ln \left(1+\frac{\theta_{r}}{2}\right)}{\theta_{r}^{2}}+\frac{\left(\theta_{r}-1\right)\left(r_{2}-r_{1}\right)}{2 \theta_{r}}+\frac{\left(r_{2}-r_{1}\right) \ln \left(1+\frac{\theta_{r}}{2}\right)}{\theta_{r}^{2}} \\
& -\frac{\left(\theta_{r}-1\right)\left(r_{4}-r_{3}\right)}{2 \theta_{r}}-\frac{\left(r_{4}-r_{3}\right) \ln \left(1+\frac{\theta_{r}}{2}\right)}{\theta_{r}^{2}}-\frac{\left(1+\theta_{r}\right)\left(r_{4}-r_{3}\right)}{2 \theta_{r}}+\frac{\left(1+\theta_{r}\right)\left(r_{4}-r_{3}\right) \ln \left(1+\frac{\theta_{r}}{2}\right)}{\theta_{r}^{2}} \\
= & \frac{r_{1}+r_{4}}{2}-\frac{\left(r_{1}-r_{2}-r_{3}+r_{4}\right) \ln \left(1+\frac{\theta_{r}}{2}\right)}{2 \theta_{r}}
\end{array}\right]
$$

which is the expectation formula of the reduction of type-2 trapezoidal fuzzy variable $\tilde{\xi}=\left(r_{1}, r_{2}, r_{3}, r_{4}, \theta_{l}, \theta_{r}\right)$ obtained by the optimistic critical value reduction method.

If $r_{2}=r_{3}$, then

$$
E\left[\tilde{\xi}_{1}\right]=\frac{r_{1}+r_{4}}{2}-\frac{\left(r_{1}-2 r_{2}+r_{4}\right) \ln \left(1+\frac{\theta_{r}}{2}\right)}{2 \theta_{r}}
$$

which is the expectation formula of the reduction of type-2 triangular fuzzy variable $\tilde{\tilde{\xi}}=$ $\left(r_{1}, r_{2}, r_{4}, \theta_{l}, \theta_{r}\right)$ obtained by the optimistic critical value reduction method.

As $\theta_{r} \rightarrow 0$, the above result (21) is converted into

$$
\lim _{\theta_{r} \rightarrow 0} E\left[\tilde{\xi}_{1}\right]=\lim _{\theta_{r} \rightarrow 0}\left[\frac{r_{1}+r_{4}}{2}-\frac{\left(r_{1}-r_{2}-r_{3}+r_{4}\right) \ln \left(1+\frac{\theta_{r}}{2}\right)}{2 \theta_{r}}\right]=\frac{r_{1}+r_{2}+r_{3}+r_{4}}{4}
$$

which is the expected value of type-1 trapezoidal fuzzy variable.

Theorem 2 Assume $\tilde{\xi_{2}}$ be the reduction of the type-2 trapezoidal fuzzy variable $\tilde{\tilde{\xi}}=$ $\left(r_{1}, r_{2}, r_{3}, r_{4}, \theta_{l}, \theta_{r}\right)$, obtained by the pessimistic critical value reduction method. Then

$$
E\left[\tilde{\xi}_{2}\right]=\frac{r_{2}+r_{3}}{2}+\frac{\left(r_{1}-r_{2}-r_{3}+r_{4}\right) \ln \left(1+\frac{\theta_{l}}{2}\right)}{2 \theta_{l}}
$$


Proof As in Theorem 1, according to Eq. (15), for a trapezoidal fuzzy variable, its possibility distribution function $\tilde{\xi_{2}}$ is given by

$$
\begin{aligned}
& \mu_{\tilde{\xi}_{2}}(x)=\operatorname{Pos}\left\{\tilde{\xi}_{2}=x\right\} \\
& \Rightarrow \mu_{\tilde{\xi}_{2}}(x)=\left\{\begin{array}{lll}
\frac{\frac{x-r_{1}}{r_{2}-r_{1}}}{1+\frac{x-r_{1}}{r_{2}-r_{1}}-\frac{x-r_{1}}{r_{2}-r_{1}}+\theta_{l} \min \left\{\frac{x-r_{1}}{r_{2}-r_{1}}, \frac{r_{2}-x}{r_{2}-r_{1}}\right\}}, & \text { if } & x \in\left[r_{1}, r_{2}\right] \\
1, & \text { if } & x \in\left(r_{2}, r_{3}\right] \\
\frac{\frac{r_{4}-x}{r_{4}-r_{3}}}{1+\frac{r_{4}-x}{r_{4}-r_{3}}-\frac{r_{4}-x}{r_{4}-r_{3}}+\theta_{l} \min \left\{\frac{r_{4}-x}{r_{4}-r_{3}}, \frac{x-r_{3}}{r_{4}-r_{3}}\right\}}, & \text { if } & x \in\left(r_{3}, r_{4}\right]
\end{array}\right. \\
& \Rightarrow \mu_{\tilde{\xi}_{2}}(x)= \begin{cases}\frac{x-r_{1}}{r_{2}-r_{1}+\theta_{l}\left(x-r_{1}\right)}, & \text { if } \quad x \in\left[r_{1}, \frac{r_{1}+r_{2}}{2}\right] \\
\frac{x-r_{1}}{r_{2}-r_{1}+\theta_{l}\left(r_{2}-x\right)}, & \text { if } \quad x \in\left(\frac{r_{1}+r_{2}}{2}, r_{2}\right] \\
1, & \text { if } x \in\left(r_{2}, r_{3}\right] \\
\frac{r_{4}-x}{r_{4}-r_{3}+\theta_{l}\left(x-r_{3}\right)}, & \text { if } \quad x \in\left(r_{3}, \frac{r_{3}+r_{4}}{2}\right] \\
\frac{r_{4}-x}{r_{4}-r_{3}+\theta_{l}\left(r_{4}-x\right)}, & \text { if } \quad x \in\left(\frac{r_{3}+r_{4}}{2}, r_{4}\right]\end{cases}
\end{aligned}
$$

Therefore the credibility of $\tilde{\xi}_{2}$ is defined by

$$
\operatorname{Cr}\left\{\tilde{\xi}_{2} \geq r\right\}=\left\{\begin{array}{lll}
1, & \text { if } & r<r_{1} \\
1-\frac{r-r_{1}}{2\left(r_{2}-r_{1}+\theta_{l}\left(r-r_{1}\right)\right)}, & \text { if } & r \in\left[r_{1}, \frac{r_{1}+r_{2}}{2}\right] \\
1-\frac{r-r_{1}}{2\left(r_{2}-r_{1}+\theta_{l}\left(r_{2}-r\right)\right)}, & \text { if } & r \in\left(\frac{r_{1}+r_{2}}{2}, r_{2}\right] \\
\frac{1}{2}, & \text { if } r \in\left(r_{2}, r_{3}\right] \\
\frac{r_{4}-r}{2\left(r_{4}-r_{3}+\theta_{l}\left(r-r_{3}\right)\right)}, & \text { if } r \in\left(r_{3}, \frac{r_{3}+r_{4}}{2}\right] \\
\frac{r_{4}-r}{2\left(r_{4}-r_{3}+\theta_{l}\left(r_{4}-r\right)\right)}, & \text { if } r \in\left(\frac{r_{3}+r_{4}}{2}, r_{4}\right] \\
0, & \text { if } r>r_{4}
\end{array}\right.
$$

and

$$
\operatorname{Cr}\left\{\tilde{\xi}_{2} \leq r\right\}=\left\{\begin{array}{lll}
0, & \text { if } & r<r_{1} \\
\frac{r-r_{1}}{2\left(r_{2}-r_{1}+\theta_{l}\left(r-r_{1}\right)\right)}, & \text { if } \quad r \in\left[r_{1}, \frac{r_{1}+r_{2}}{2}\right] \\
\frac{r-r_{1}}{2\left(r_{2}-r_{1}+\theta_{l}\left(r_{2}-r\right)\right)}, & \text { if } \quad r \in\left(\frac{r_{1}+r_{2}}{2}, r_{2}\right] \\
\frac{1}{2}, & \text { if } r \in\left(r_{2}, r_{3}\right] \\
1-\frac{r_{4}-r}{2\left(r_{4}-r_{3}+\theta_{l}\left(r-r_{3}\right)\right)}, & \text { if } & r \in\left(r_{3}, \frac{r_{3}+r_{4}}{2}\right] \\
1-\frac{r_{4}-r}{2\left(r_{4}-r_{3}+\theta_{l}\left(r_{4}-r\right)\right)}, & \text { if } & r \in\left(\frac{r_{3}+r_{4}}{2}, r_{4}\right] \\
1, & \text { if } \quad r>r_{4}
\end{array}\right.
$$

If $r_{4} \leq 0$, then we have

$$
\begin{aligned}
E\left[\tilde{\xi}_{2}\right]= & \int_{0}^{\infty} \operatorname{Cr}\left\{\tilde{\xi}_{2} \geq r\right\} d r-\int_{-\infty}^{0} \operatorname{Cr}\left\{\tilde{\xi}_{2} \leq r\right\} d r \\
= & -\int_{r_{1}}^{\frac{r_{1}+r_{2}}{2}} \frac{r-r_{1}}{2\left(r_{2}-r_{1}+\theta_{l}\left(r-r_{1}\right)\right)} d r-\int_{\frac{r_{1}+r_{2}}{2}}^{r_{2}} \frac{r-r_{1}}{2\left(r_{2}-r_{1}+\theta_{l}\left(r_{2}-r\right)\right)} d r \\
& -\int_{r_{2}}^{r_{3}} \frac{1}{2} d r-\int_{r_{3}}^{\frac{r_{3}+r_{4}}{2}} 1 d r+\int_{r_{3}}^{\frac{r_{3}+r_{4}}{2}} \frac{r_{4}-r}{2\left(r_{4}-r_{3}+\theta_{l}\left(r-r_{3}\right)\right)} d r \\
& -\int_{\frac{r_{3}+r_{4}}{2}}^{r_{4}} 1 d r+\int_{\frac{r_{3}+r_{4}}{2}}^{r_{4}} \frac{r_{4}-r}{2\left(r_{4}-r_{3}+\theta_{l}\left(r_{4}-r\right)\right)} d r-\int_{r_{4}}^{0} 1 d r \\
= & \frac{r_{2}+r_{3}}{2}+\frac{\left(r_{1}-r_{2}-r_{3}+r_{4}\right) \ln \left(1+\frac{\theta_{l}}{2}\right)}{2 \theta_{l}}
\end{aligned}
$$

which is the expectation formula of the reduction of type- 2 trapezoidal fuzzy variable $\tilde{\tilde{\xi}}=\left(r_{1}, r_{2}, r_{3}, r_{4}, \theta_{l}, \theta_{r}\right)$ obtained by the pessimistic critical value reduction method. 
If $r_{2}=r_{3}$, then

$$
E\left[\tilde{\xi}_{2}\right]=r_{2}+\frac{\left(r_{1}-2 r_{2}+r_{4}\right) \ln \left(1+\frac{\theta_{l}}{2}\right)}{2 \theta_{l}}
$$

which is the expectation formula of the reduction of type-2 triangular fuzzy variable $\tilde{\tilde{\xi}}=$ $\left(r_{1}, r_{2}, r_{4}, \theta_{l}, \theta_{r}\right)$ obtained by the pessimistic critical value reduction method.

As $\theta_{l} \rightarrow 0$, the above result (28) is converted into

$$
\lim _{\theta_{l} \rightarrow 0} E\left[\tilde{\xi}_{2}\right]=\lim _{\theta_{l} \rightarrow 0}\left[\frac{r_{2}+r_{3}}{2}+\frac{\left(r_{1}-r_{2}-r_{3}+r_{4}\right) \ln \left(1+\frac{\theta_{l}}{2}\right)}{2 \theta_{l}}\right]=\frac{r_{1}+r_{2}+r_{3}+r_{4}}{4}
$$

which is the expected value of type-1 trapezoidal fuzzy variable.

Theorem 3 Assume $\tilde{\xi}_{3}$ to be the reduction of the type-2 trapezoidal fuzzy variable $\tilde{\tilde{\xi}}=$ $\left(r_{1}, r_{2}, r_{3}, r_{4}, \theta_{l}, \theta_{r}\right)$, obtained by the critical value reduction method. Then

$$
\begin{aligned}
E\left[\tilde{\xi}_{3}\right]= & \frac{r_{1}+r_{2}+r_{3}+r_{4}}{4}+\frac{r_{1}-r_{2}-r_{3}+r_{4}}{8}\left[\frac{1}{\theta_{r}}-\frac{1}{\theta_{l}}-\frac{\left(1+\theta_{r}\right) \ln \left(1+\theta_{r}\right)}{\theta_{r}^{2}}+\right. \\
& \left.\frac{\left(1+\theta_{l}\right) \ln \left(1+\theta_{l}\right)}{\theta_{l}^{2}}\right]
\end{aligned}
$$

Proof From Eq. (16), we have, the possibility distribution of a trapezoidal fuzzy variable $\tilde{\xi}_{3}$ is given by

$$
\begin{aligned}
& \mu_{\tilde{\xi}_{3}}(x)=\operatorname{Pos}\left\{\tilde{\xi}_{3}=x\right\}
\end{aligned}
$$

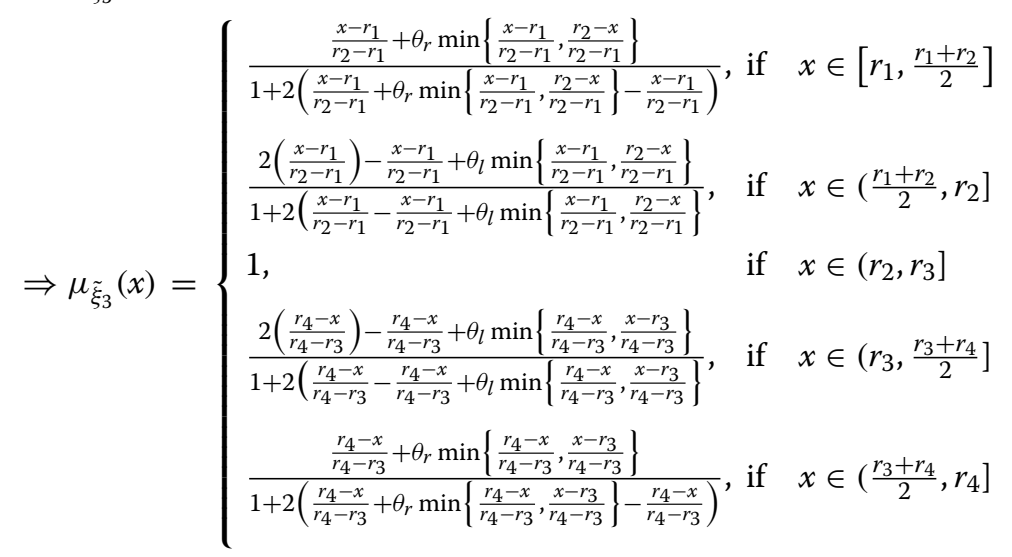

$$
\begin{aligned}
& \Rightarrow \mu_{\tilde{\xi}_{3}}(x)=\left\{\begin{array}{lll}
\frac{\left(1+\theta_{r}\right)\left(x-r_{1}\right)}{r_{2}-r_{1}+2 \theta_{r}\left(x-r_{1}\right)}, & \text { if } & x \in\left[r_{1}, \frac{r_{1}+r_{2}}{2}\right] \\
\frac{\left(1-\theta_{l}\right) x+\theta_{l} r_{2}-r_{1}}{r_{2}-r_{1}+2 \theta_{l}\left(r_{2}-x\right)}, & \text { if } & x \in\left(\frac{r_{1}+r_{2}}{2}, r_{2}\right] \\
1, & \text { if } & x \in\left(r_{2}, r_{3}\right] \\
\frac{\left(\theta_{l}-1\right) x-\theta_{l} r_{3}+r_{4}}{r_{4}-r_{3}+2 \theta_{l}\left(x-r_{3}\right)}, & \text { if } & x \in\left(r_{3}, \frac{r_{3}+r_{4}}{2}\right] \\
\frac{\left(1+\theta_{r}\right)\left(r_{4}-x\right)}{r_{4}-r_{3}+2 \theta_{r}\left(r_{4}-x\right)}, & \text { if } & x \in\left(\frac{r_{3}+r_{4}}{2}, r_{4}\right]
\end{array}\right.
\end{aligned}
$$


Therefore the credibility of $\tilde{\xi}_{3}$ is defined by

$$
C r\left\{\tilde{\xi}_{3} \geq r\right\}=\left\{\begin{array}{lll}
1, & \text { if } & r<r_{1} \\
1-\frac{\left(1+\theta_{r}\right)\left(r-r_{1}\right)}{2\left(r_{2}-r_{1}+2 \theta_{r}\left(r-r_{1}\right)\right)}, & \text { if } & r \in\left[r_{1}, \frac{r_{1}+r_{2}}{2}\right] \\
1-\frac{\left(1-\theta_{l}\right) r+l}{2\left(r_{2}-r_{2}-r_{1}+2 \theta_{l}\left(r_{2}-r\right)\right)}, & \text { if } & r \in\left(\frac{r_{1}+r_{2}}{2}, r_{2}\right] \\
\frac{1}{2}, & \text { if } & r \in\left(r_{2}, r_{3}\right] \\
\frac{\left(\theta_{l}-1\right) r-\theta_{l} r_{3}+r_{4}}{2\left(r_{4}-r_{3}+2 l_{l}\left(r-r_{3}\right)\right)}, & \text { if } & r \in\left(r_{3}, \frac{r_{3}+r_{4}}{2}\right] \\
\frac{\left(1+\theta_{r}\right)\left(r_{4}-r\right)}{2\left(r_{4}-r_{3}+2 \theta_{r}\left(r_{4}-r\right)\right)}, & \text { if } & r \in\left(\frac{r_{3}+r_{4}}{2}, r_{4}\right] \\
0, & \text { if } & r>r_{4}
\end{array}\right.
$$

and

$$
\operatorname{Cr}\left\{\tilde{\xi}_{3} \leq r\right\}=\left\{\begin{array}{lll}
0, & \text { if } & r<r_{1} \\
\frac{\left(1+\theta_{r}\right)\left(r-r_{1}\right)}{2\left(r_{2}-r_{1}+2+r_{r}\left(r-r_{1}\right)\right)}, & \text { if } & r \in\left[r_{1}, \frac{r_{1}+r_{2}}{2}\right] \\
\frac{\left(1-\theta_{l}\right) r+\theta_{l}\left(r_{2}-r_{1}\right.}{2\left(r_{2}-r_{1}+2 \theta_{l}\left(r_{2}-r\right)\right)}, & \text { if } & r \in\left(\frac{r_{1}+r_{2}}{2}, r_{2}\right] \\
\frac{1}{2}, & \text { if } & r \in\left(r_{2}, r_{3}\right] \\
1-\frac{\left(\theta_{l}-1\right) r-\theta_{l} r_{3}+r_{4}}{2\left(r_{4}-r_{3}+2 \theta_{l}\left(r-r_{3}\right)\right)}, & \text { if } & r \in\left(r_{3}, \frac{r_{3}+r_{4}}{2}\right] \\
1-\frac{\left(1+\theta_{r}\right)\left(r_{4}-r\right)}{2\left(r_{4}-r_{3}+2 \theta_{r}\left(r_{4}-r\right)\right)}, & \text { if } & r \in\left(\frac{r_{3}+r_{4}}{2}, r_{4}\right] \\
1, & \text { if } & r>r_{4}
\end{array}\right.
$$

If $r_{1} \leq 0 \leq \frac{r_{1}+r_{2}}{2}$, then we have

$$
\begin{aligned}
E\left[\tilde{\xi}_{3}\right]= & \int_{0}^{\infty} C r\left\{\tilde{\xi}_{3} \geq r\right\} d r-\int_{-\infty}^{0} C r\left\{\tilde{\xi}_{3} \leq r\right\} d r \\
= & \int_{0}^{\frac{r_{1}+r_{2}}{2}} 1 d r-\int_{0}^{\frac{r_{1}+r_{2}}{2}} \frac{\left(1+\theta_{r}\right)\left(r-r_{1}\right)}{2\left(r_{2}-r_{1}+2 \theta_{r}\left(r-r_{1}\right)\right)} d r+\int_{\frac{r_{1}+r_{2}}{2}}^{r_{2}} 1 d r-\int_{\frac{r_{1}+r_{2}}{2}}^{r_{2}} \frac{\left(1-\theta_{l}\right) r+\theta_{l} r_{2}-r_{1}}{2\left(r_{2}-r_{1}+2 \theta_{l}\left(r_{2}-r\right)\right)} d r \\
& +\int_{r_{2}}^{r_{3}} \frac{1}{2} d r-\int_{r_{3}}^{\frac{r_{3}+r_{4}}{2}} \frac{\left(\theta_{l}-1\right) r-\theta_{l} r_{3}+r 4}{2\left(r_{4}-r_{3}+2 \theta_{l}\left(r-r_{3}\right)\right)} d r+\int_{\frac{r_{3}+r_{4}}{2}}^{r_{4}} \frac{\left(1+\theta_{r}\right)\left(r_{4}-r\right)}{2\left(r_{4}-r_{3}+2 \theta_{r}\left(r_{4}-r\right)\right)} d r \\
& -\int_{r_{1}}^{0} \frac{\left(1+\theta_{r}\right)\left(r-r_{1}\right)}{2\left(r_{2}-r_{1}+2 \theta_{r}\left(r-r_{1}\right)\right)} d r \\
= & \frac{r_{1}+r_{2}+r_{3}+r_{4}}{4}+\frac{r_{1}-r_{2}-r_{3}+r_{4}}{8}\left[\frac{1}{\theta_{r}}-\frac{1}{\theta_{l}}-\frac{\left(1+\theta_{r}\right) \ln \left(1+\theta_{r}\right)}{\theta_{r}^{2}}+\frac{\left(1+\theta_{l}\right) \ln \left(1+\theta_{l}\right)}{\theta_{l}^{2}}\right]
\end{aligned}
$$

which is the expectation formula of the reduction of type-2 triangular fuzzy variable $\tilde{\tilde{\xi}}=$ $\left(r_{1}, r_{2}, r_{3}, r_{4}, \theta_{l}, \theta_{r}\right)$ obtained by the critical value reduction method.

If $r_{2}=r_{3}$, then

$E\left[\tilde{\xi}_{3}\right]=\frac{r_{1}+2 r_{2}+r_{4}}{4}+\frac{r_{1}-2 r_{2}+r_{4}}{8}\left[\frac{1}{\theta_{r}}-\frac{1}{\theta_{l}}-\frac{\left(1+\theta_{r}\right) \ln \left(1+\theta_{r}\right)}{\theta_{r}^{2}}+\frac{\left(1+\theta_{l}\right) \ln \left(1+\theta_{l}\right)}{\theta_{l}^{2}}\right]$

which is the expectation formula of the reduction of type- 2 triangular fuzzy variable $\tilde{\tilde{\xi}}=$ $\left(r_{1}, r_{2}, r_{4}, \theta_{l}, \theta_{r}\right)$ obtained by the critical value reduction method. 
As $\theta_{l}$ and $\theta_{r} \rightarrow 0$, the above Eq. (35) is converted into

$$
\begin{aligned}
\lim _{\theta_{l}, \theta_{r} \rightarrow 0} E\left[\tilde{\xi}_{3}\right] & =\lim _{\theta_{l}, \theta_{r} \rightarrow 0} \frac{r_{1}+2 r_{2}+r_{3}}{4}+\frac{r_{1}+2 r_{2}+r_{3}}{8} \\
& \times\left[\frac{1}{\theta_{r}}-\frac{1}{\theta_{l}}-\frac{\left(1+\theta_{r}\right) \ln \left(1+\theta_{r}\right)}{\theta_{r}^{2}}+\frac{\left(1+\theta_{l}\right) \ln \left(1+\theta_{l}\right)}{\theta_{l}^{2}}\right] \\
& \approx \frac{r_{1}+r_{2}+r_{3}+r_{4}}{4}
\end{aligned}
$$

which is the expected value of type-1 trapezoidal fuzzy variable.

Example 1 Let $\tilde{\tilde{\xi}}=(1,3,5,6,0.5,1)$ be a T2 trapezoidal fuzzy variable, $\tilde{\xi}_{1}$ be the reduction of $\tilde{\tilde{\xi}}$ with the optimistic critical value reduction method, $\tilde{\xi}_{2}$ be the reduction of $\tilde{\tilde{\xi}}$ with the pessimistic critical value reduction method and $\tilde{\xi}_{3}$ be the reduction of $\tilde{\tilde{\xi}}$ with the critical value reduction method. Then,

$$
\begin{aligned}
E\left[\tilde{\xi}_{1}\right] & =\frac{7}{2}+\frac{1}{2} \ln \left(\frac{3}{2}\right)=3.703 \\
E\left[\tilde{\xi}_{2}\right] & =4-\ln \left(\frac{5}{4}\right)=3.78 \\
\text { and } E\left[\tilde{\xi}_{3}\right] & =\frac{31}{8}+\frac{1}{4} \ln (2)-\frac{3}{4} \ln \left(\frac{3}{2}\right)=3.74
\end{aligned}
$$

\section{Fuzzy goal programming method}

Sakawa [15] proposed the fuzzy goal programming technique (GPT) to solve linear and nonlinear multi-objective programming problems (MOPPs). The MOPPs can be taken as

$$
\left\{\begin{array}{l}
\max _{x}\left[f_{1}(x), f_{2}(x), \cdots, f_{m}(x)\right] \\
\{x \in X .
\end{array}\right.
$$

Let us consider that decision makers have fixed the membership function $\mu_{k}\left(f_{k}(x)\right)$ and given the goal membership function value $(k=1,2, \cdots, m)$. We assume the following programming problem as

$$
\left\{\begin{array}{l}
\max _{x} \sum_{i=1}^{m} d_{i}^{-} \\
\left\{\begin{array}{l}
\mu_{k}\left(f_{k}(x)\right)+d_{i}^{+}-d_{i}^{-}=\bar{\mu}_{k} \\
x \in X \\
d_{i}^{+} d_{i}^{-}=0, d_{i}^{+}, d_{i}^{-} \geq 0, k=0,1,2, \cdots, m .
\end{array}\right.
\end{array}\right.
$$

where $d_{i}^{+}, d_{i}^{-}$denotes the positive and negative deviations.

The above single objective problem is then solved using soft computing technique.

\section{Notations}

In this investigation, a single stage solid transportation problem (STP) consisting of vendors and customers are considered. Here, products from each vendor can be transported to each customer by means of each conveyance. The STP is formulated as a multiobjective optimization problem, where the objectives are minimization of transportation cost and minimization of total time of transportation. The following notations are used:

(i) $M=$ number of origins/vendors from which the products are shipped (indexed $i=1,2, \cdots, M)$. 
(ii) $N=$ number of destinations/customers to which the products are shipped (indexed $j=1,2, \cdots, N)$.

(iii) $K=$ number of conveyances (indexed $k=1,2, \cdots, K$ ).

(iv) $\tilde{\tilde{c}}_{i j k}=$ unit transportation cost for the product from $\mathrm{i}$-th manufacturer to $\mathrm{j}$-th destination by means of $\mathrm{k}$-th conveyance, which are T2TrFVs in nature( $\$ /$ ton).

(v) $x_{i j k}=$ the amount of product(tons) to be transported from $\mathrm{i}$-th origin to $\mathrm{j}$-th destination by means of $\mathrm{k}$-th conveyance (decision variables)

(vi) $\quad \tilde{\tilde{a}}_{i}=$ availability at $\mathrm{i}$-th origin, which are T2TrFVs in nature (tons).

(vii) $\tilde{\tilde{b}}_{j}=$ demand at $\mathrm{j}$-th destination, which are T2TrFVs in nature(tons).

(viii) $\tilde{\tilde{e}}_{k}=$ capacity at $\mathrm{k}$-th conveyance, which are T2TrFVs in nature(tons).

(ix) $\tilde{\tilde{t}}_{i j k}=$ unit transportation time from $\mathrm{i}$-th origin to $\mathrm{j}$-th destination by means of $\mathrm{k}$-th conveyance, which are T2TrFVs in nature(hrs/ton)

(x) $y_{i j k}$ are binary variables, defined by

$$
y_{i j k}=\left\{\begin{array}{l}
1, x_{i j k} \neq 0 \\
0, x_{i j k}=0
\end{array}\right.
$$

(xi) $\tilde{\tilde{d}}_{i j k}=$ unit loading and unloading time at $\mathrm{i}$-th origin and $\mathrm{j}$-th destination, respectively, when transported by means of $k$-th conveyance, which are T2TrFVs in nature(hrs/ton)

(xii) $\tilde{\tilde{f}}_{1}=$ total transportation cost of the problem (\$).

(x) $\tilde{\tilde{f}}_{2}=$ total transportation time of the problem (h).

\section{Formulation of type $\mathbf{2}$ fuzzy solid transportation problem (T2FSTP)}

In this model, we minimize total transportation cost $\tilde{\tilde{f}}_{1}$ and the total transportation time $\tilde{\tilde{f}}_{2}$ over a distribution system consisting of vendors and customers. The T2FSTP is formulated as

$$
\begin{aligned}
\min \tilde{\tilde{f}}_{1}= & \sum_{i=1}^{M} \sum_{j=1}^{N} \sum_{k=1}^{K}\left\{\tilde{\widetilde{c}}_{i j k} \cdot x_{i j k}\right\} \\
\min \tilde{\tilde{f}}_{2}= & \sum_{i=1}^{M} \sum_{j=1}^{N} \sum_{k=1}^{K}\left\{\tilde{\tilde{t}}_{i j k} \cdot y_{i j k}+\tilde{\tilde{d}}_{i j k} \cdot x_{i j k}\right\} \\
\operatorname{s.t} \quad & \begin{cases}\sum_{j=1}^{N} \sum_{k=1}^{K} x_{i j k} \leq \widetilde{\tilde{a}}_{i}, & i=1,2,3 \cdots, M \\
\sum_{i=1}^{M} \sum_{k=1}^{K} x_{i j k} \geq \widetilde{\widetilde{b}}_{j}, & j=1,2,3 \cdots, N \\
\sum_{i=1}^{M} \sum_{j=1}^{N} x_{i j k} \leq \widetilde{\tilde{e}}_{k}, & k=1,2,3 \cdots, K \\
x_{i j k} \geq 0, & \forall i, j, k\end{cases}
\end{aligned}
$$

For the objective function $\tilde{\tilde{f}}_{1}$, we have, unit transportation costs, $\tilde{\tilde{c}}_{i j k}=$ $\left(c_{i j k}^{1}, c_{i j k}^{2}, c_{i j k}^{3}, c_{i j k}^{4}, c_{i j k}^{\theta_{l}}, c_{i j k}^{\theta_{r}}\right)$, and for the objective function $\tilde{\tilde{f}}_{2}$, we have, unit transportation 
time $\tilde{\tilde{t}}_{i j k}=\left(t_{i j k}^{1}, t_{i j k}^{2}, t_{i j k}^{3}, t_{i j k}^{4}, t_{i j k}^{\theta_{l}}, t_{i j k}^{\theta_{r}}\right)$, unit loading and unloading time $\tilde{\tilde{d}}_{i j k}=$ $\left(d_{i j k}^{1}, d_{i j k}^{2}, d_{i j k}^{3}, d_{i j k}^{4}, d_{i j k}^{\theta_{l}}, d_{i j k}^{\theta_{r}}\right)$ and for the constraints we have, availability of product $\tilde{\tilde{a}}_{i}=$ $\left(a_{i}^{1}, a_{i}^{2}, a_{i}^{3}, a_{i}^{4}, a_{i}^{\theta_{l}}, a_{i}^{\theta_{r}}\right)$, demand of product $\tilde{\tilde{b}}_{j}=\left(b_{j}^{1}, b_{j}^{2}, b_{j}^{3}, b_{j}^{4}, b_{j}^{\theta_{l}}, b_{j}^{\theta_{r}}\right)$,

capacity of conveyance $\tilde{\tilde{e}}_{k}=\left(e_{k}^{1}, e_{k}^{2}, e_{k}^{3}, e_{k}^{4}, e_{k}^{\theta_{l}}, e_{k}^{\theta_{r}}\right)$,

\section{Equivalent crisp problem in $\mathrm{T} 2$}

The STP under T2TrFV environment is now being reduced to an STP in non-fuzzy environment by applying the expectation formulas.

\section{Optimistic value}

Using Theorem 1, and the Eqs. (40)-(42), we have obtained the equivalent crisp problem for optimistic values as

$$
\min f_{1}=\sum_{i=1}^{M} \sum_{j=1}^{N} \sum_{k=1}^{K}\left[\left\{\frac{c_{i j k}^{1}+c_{i j k}^{4}}{2}-\frac{\left(c_{i j k}^{1}-c_{i j k}^{2}-c_{i j k}^{3}+c_{i j k}^{4}\right) \ln \left(1+\frac{c_{i j k}^{\theta_{r}}}{2}\right)}{2 c_{i j k}^{\theta_{r}}}\right\} \cdot x_{i j k}\right]
$$

$$
\begin{aligned}
& \min f_{2}=\sum_{i=1}^{M} \sum_{j=1}^{N} \sum_{k=1}^{K}\left[\left\{\frac{t_{i j k}^{1}+t_{i j k}^{4}}{2}-\frac{\left(t_{i j k}^{1}-t_{i j k}^{2}-t_{i j k}^{3}+t_{i j k}^{4}\right) \ln \left(1+\frac{t_{i j k}^{\theta_{r}}}{2}\right)}{2 t_{i j k}^{\theta_{r}}}\right\} \cdot y_{i j k}\right. \\
& \left.+\left\{\frac{d_{i j k}^{1}+d_{i j k}^{4}}{2}-\frac{\left(d_{i j k}^{1}-d_{i j k}^{2}-d_{i j k}^{3}+d_{i j k}^{4}\right) \ln \left(1+\frac{d_{i j k}^{\theta_{r}}}{2}\right)}{2 d_{i j k}^{\theta_{r}}}\right\} \cdot x_{i j k}\right]
\end{aligned}
$$

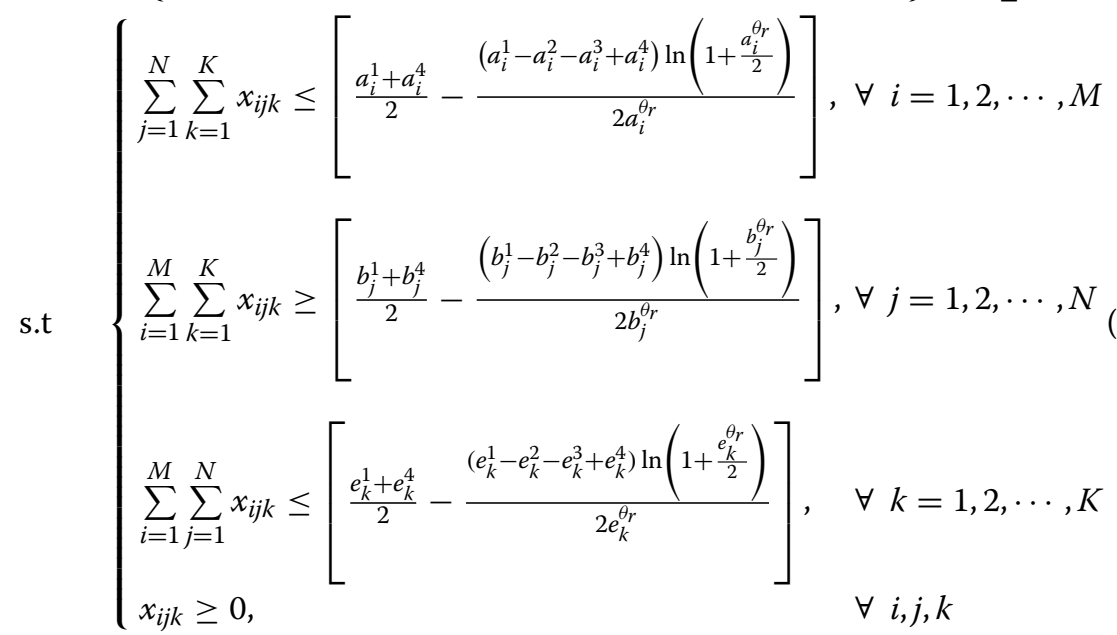




\section{Pessimistic value}

Using Theorem 2, and the Eqs. (40)-(42), we have obtained the equivalent crisp problem for pessimistic values as

$$
\min f_{1}=\sum_{i=1}^{M} \sum_{j=1}^{N} \sum_{k=1}^{K}\left[\left\{\frac{c_{i j k}^{2}+c_{i j k}^{3}}{2}+\frac{\left(c_{i j k}^{1}-c_{i j k}^{2}-c_{i j k}^{3}+c_{i j k}^{4}\right) \ln \left(1+\frac{c^{\theta_{l}}}{2}\right)}{2 c_{i j k}^{\theta_{l}}}\right\} \cdot x_{i j k}\right]
$$

$$
\begin{aligned}
& \min f_{2}=\sum_{i=1}^{M} \sum_{j=1}^{N} \sum_{k=1}^{K}\left[\left\{\frac{t_{i j k}^{2}+t_{i j k}^{3}}{2}+\frac{\left(t_{i j k}^{1}-t_{i j k}^{2}-t_{i j k}^{3}+t_{i j k}^{4}\right) \ln \left(1+\frac{t_{i j k}^{\theta_{l}}}{2}\right)}{2 t_{i j k}^{\theta_{l}}}\right\} \cdot y_{i j k}\right. \\
& \left.+\left\{\frac{d_{i j k}^{2}+d_{i j k}^{3}}{2}+\frac{\left(d_{i j k}^{1}-d_{i j k}^{2}-d_{i j k}^{3}+d_{i j k}^{4}\right) \ln \left(1+\frac{d_{i j k}^{\theta_{l}}}{2}\right)}{2 d_{i j k}^{\theta_{l}}}\right\} \cdot x_{i j k}\right]
\end{aligned}
$$

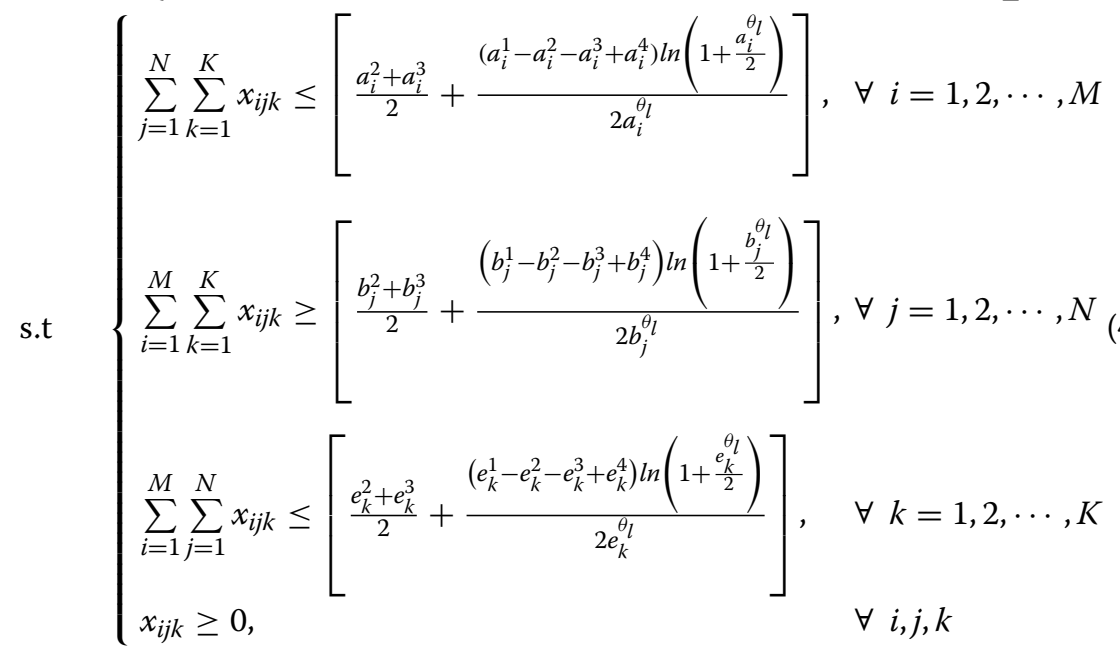

\section{CV value}

Using Theorem 3, and the Eqs. (40)-(42), we have obtained the equivalent crisp problem for CV values as:

$$
\begin{aligned}
\min f_{1}= & \sum_{i=1}^{M} \sum_{j=1}^{N} \sum_{k=1}^{K}\left[\left\{\frac{c_{i j k}^{1}+c_{i j k}^{2}+c_{i j k}^{3}+c_{i j k}^{4}}{4}+\frac{c_{i j k}^{1}-c_{i j k}^{2}-c_{i j k}^{3}+c_{i j k}^{4}}{8}\right.\right. \\
& \left.\left.\left\{\frac{1}{c_{i j k}^{\theta_{r}}}-\frac{1}{c_{i j k}^{\theta_{l}}}-\frac{\left(1+c_{i j k}^{\theta_{r}}\right) \ln \left(1+c_{i j k}^{\theta_{r}}\right)}{c_{i j k}^{\theta_{r}^{2}}}+\frac{\left(1+c_{i j k}^{\theta_{l}}\right) \ln \left(1+c_{i j k}^{\theta_{l}}\right)}{c_{l}^{2}}\right\}\right\} \cdot x_{i j k}\right]
\end{aligned}
$$




$$
\begin{aligned}
\min f_{2}= & \sum_{i=1}^{M} \sum_{j=1}^{N} \sum_{k=1}^{K}\left[\left\{\frac{t_{i j k}^{1}+t_{i j k}^{2}+t_{i j k}^{3}+t_{i j k}^{4}}{4}+\frac{t_{i j k}^{1}-t_{i j k}^{2}+t_{i j k}^{3}+t_{i j k}^{4}}{8}\left[\frac{1}{t_{i j k}^{\theta_{r}}}-\frac{1}{t_{i j k}^{\theta_{l}}}-\frac{\left(1+t_{i j k}^{\theta_{r}}\right) \ln \left(1+t_{i j k}^{\theta_{r}}\right)}{t_{i j k}^{\theta_{r}^{2}}}\right.\right.\right. \\
& \left.\left.+\frac{\left(1+t_{i j k}^{\theta_{l}}\right) \ln \left(1+t_{i j k}^{\theta_{l}}\right)}{t_{i j k}^{\theta_{l}^{2}}}\right]\right\} \cdot y_{i j k}+\left\{\frac{t_{i j k}^{1}+t_{i j k}^{2}+t_{i j k}^{3}+t_{i j k}^{4}}{4}+\frac{t_{i j k}^{1}-t_{i j k}^{2}+t_{i j k}^{3}+t_{i j k}^{4}}{8}\left[\frac{1}{t_{i j k}^{\theta_{r}}}\right.\right. \\
& \left.\left.\left.-\frac{1}{t_{i j k}^{\theta_{l}}}-\frac{\left(1+t_{i j k}^{\theta_{r}}\right) \ln \left(1+t_{i j k}^{\theta_{r}}\right)}{t_{i j k}^{\theta_{r}^{2}}}+\frac{\left(1+t_{i j k}^{\theta_{l}}\right) \ln \left(1+t_{i j k}^{\theta_{l}}\right)}{t_{i j k}^{\theta_{l}^{2}}}\right]\right\} \cdot x_{i j k}\right]
\end{aligned}
$$

$$
\mathrm{s.t} \begin{cases}\sum_{j=1}^{N} \sum_{k=1}^{K} x_{i j k} \leq\left[\frac{a_{i}^{1}+a_{i}^{2}+a_{i}^{3}+a_{i}^{4}}{4}+\frac{a_{i}^{1}-a_{i}^{2}+a_{i}^{3}+a_{i}^{4}}{8}\left\{\frac{1}{a_{i}^{\theta_{r}}}-\frac{1}{a_{i}^{\theta_{l}}}-\frac{\left(1+a_{i}^{\theta_{r}}\right) \ln \left(1+a_{i}^{\theta_{r}}\right)}{a_{i}^{\theta_{r}^{2}}}\right.\right. & \\ \left.\left.+\frac{\left(1+a_{i}^{\theta_{l}}\right) \ln \left(1+a_{i}^{\theta_{l}}\right)}{a_{i}^{\theta_{l}^{2}}}\right\}\right], & \forall i=1,2, \ldots, M \\ \sum_{i=1}^{M} \sum_{k=1}^{K} x_{i j k} \geq\left[\frac{b_{j}^{1}+b_{j}^{2}+b_{j}^{3}+b_{j}^{4}}{4}+\frac{b_{j}^{1}-b_{j}^{2}-b_{j}^{3}+b_{j}^{4}}{8}\left\{\frac{1}{b_{j}^{\theta_{r}}}-\frac{1}{b_{j}^{\theta_{l}}}-\frac{\left(1+b_{j}^{\theta_{r}}\right) \ln \left(1+b_{j}^{\theta_{r}}\right)}{b_{j}^{\theta_{r}^{2}}}\right.\right. & \\ \left.\left.+\frac{\left(1+b_{j}^{\theta_{l}}\right) \ln \left(1+b_{j}^{\theta_{l}}\right)}{b_{j}^{\theta_{l}^{2}}}\right\}\right], & \forall j=1,2, \ldots, N \\ \sum_{i=1}^{M} \sum_{j=1}^{N} x_{i j k} \leq\left[\frac{e_{k}^{1}+e_{k}^{2}+e_{k}^{3}+e_{k}^{4}}{4}+\frac{e_{k}^{1}-e_{k}^{2}-e_{k}^{3}+e_{k}^{4}}{8}\left\{\frac{1}{e_{k}^{\theta_{r}}}-\frac{1}{e_{k}^{\theta_{l}}}-\frac{\left(1+e_{k}^{\theta_{r}}\right) \ln \left(1+e_{k}^{\theta_{r}}\right)}{e_{k}^{\theta_{r}^{2}}}\right.\right. & \\ \left.+\frac{\left(1+e_{k}^{\theta_{l}}\right) \ln \left(1+e_{k}^{\theta_{l}}\right)}{e_{k}^{\theta_{l}^{2}}}\right\}, & \forall k=1,2, \cdots, K \\ x_{i j k} \geq 0, & \forall i, j, k\end{cases}
$$

\section{Equivalent crisp problem in $\mathrm{T} 1$}

If the input parameters are in type-1 trapezoidal fuzzy in nature, then using Eq. (23) of Theorem-1 and Eqs. (40)-(42), we have obtained the equivalent crisp problem for expected value as

$$
\begin{aligned}
& \min f_{1}=\sum_{i=1}^{M} \sum_{j=1}^{N} \sum_{k=1}^{K}\left[\left\{\frac{c_{i j k}^{1}+c_{i j k}^{2}+c_{i j k}^{3}+c_{i j k}^{4}}{4}\right\} \cdot x_{i j k}\right] \\
& \min f_{2}=\sum_{i=1}^{M} \sum_{j=1}^{N} \sum_{k=1}^{K}\left[\left\{\frac{t_{i j k}^{1}+t_{i j k}^{2}+t_{i j k}^{3}+t_{i j k}^{4}}{4}\right\} \cdot y_{i j k}+\left\{\frac{d_{i j k}^{1}+d_{i j k}^{2}+d_{i j k}^{3}+d_{i j k}^{4}}{4}\right\} \cdot x_{i j k}\right] \\
& \text { s.t } \begin{cases}\sum_{j=1}^{N} \sum_{k=1}^{K} x_{i j k} \leq\left[\frac{a_{i}^{1}+a_{i}^{2}+a_{i}^{3}+a_{i}^{4}}{4}\right], & \forall i=1,2, \cdots, M \\
\sum_{i=1}^{M} \sum_{k=1}^{K} x_{i j k} \geq\left[\frac{b_{j}^{1}+b_{j}^{2}+b_{j}^{3}+b_{j}^{4}}{4}\right], & \forall j=1,2, \cdots, N \\
\sum_{i=1}^{M} \sum_{j=1}^{N} x_{i j k} \leq\left[\frac{e_{k}^{1}+e_{k}^{2}+e_{k}^{3}+e_{k}^{4}}{4}\right], & \forall k=1,2, \cdots, K \\
x_{i j k} \geq 0, & \forall i, j, k\end{cases}
\end{aligned}
$$


Table 1 Input data for this problem

\begin{tabular}{llll}
\hline$\tilde{\tilde{a}}_{1}$ & $(28.8,30.4,34.5,37.9,0.5,1)$ & $\tilde{\tilde{b}}_{1}$ & $(12.8,14.8,16.8,17.8,0.5,1)$ \\
$\tilde{\tilde{a}}_{2}$ & $(30.9,33.9,36.9,40,0.5,1)$ & $\tilde{\tilde{b}}_{2}$ & $(14.4,16.8,18.6,21,0.5,1)$ \\
$\tilde{\tilde{c}}_{111}$ & $(7,9,11,12,0.5,1)$ & $\tilde{\tilde{c}}_{112}$ & $(5,7,9,10,0.5,1)$ \\
$\tilde{\tilde{c}}_{121}$ & $(6,7,10,12,0.5,1)$ & $\tilde{\tilde{c}}_{122}$ & $(5,8,12,14,0.5,1)$ \\
$\tilde{\tilde{c}}_{211}$ & $(6.9,8.9,10.9,10,0.5,1)$ & $\tilde{\tilde{c}}_{212}$ & $(4.9,6.9,8.9,10,0.5,1)$ \\
$\tilde{\tilde{c}}_{221}$ & $(6.9,7.9,10.9,10,0.5,1)$ & $\tilde{\tilde{c}}_{222}$ & $(5.9,8.9,12.9,10,0.5,1)$ \\
$\tilde{\tilde{t}}_{111}$ & $(4,5,7,8,0.5,1)$ & $\tilde{\tilde{c}}_{112}$ & $(6,8,10,12,0.5,1)$ \\
$\tilde{\tilde{t}}_{121}$ & $(3,4,6,7,0.5,1)$ & $\tilde{\tilde{t}}_{122}$ & $(5,6,8,9,0.5,1)$ \\
$\tilde{\tilde{t}}_{211}$ & $\tilde{\tilde{t}}_{212}$ & $(6.9,8.9,10.9,12.9,0.5,1)$ \\
$\tilde{\tilde{t}}_{221}$ & $(4.9,5.9,7.9,8.9,0.5,1)$ & $(5.9,6.9,8.9,9.9,0.5,1)$ \\
$\tilde{\tilde{d}}_{111}$ & $(3.9,4.9,6.9,7.9,0.5,1)$ & $\tilde{\tilde{t}}_{222}$ & $(1,2,4,5,0.5,1)$ \\
$\tilde{\tilde{d}}_{121}$ & $(0.8,1,2,2.5,0.5,1)$ & $(0.8,1.9,3,4.9,0.5,1)$ \\
$\tilde{\tilde{d}}_{211}$ & $(1,1.5,3.5,6,0.5,1)$ & $\tilde{\tilde{d}}_{122}$ & $(1.9,2,4.9,5.9,0.5,1)$ \\
$\tilde{\tilde{d}}_{221}$ & $(0.8,1.9,2.9,2.5,0.5,1)$ & $\tilde{\tilde{d}}_{212}$ & $(0.8,1,3,4,0.5,1)$ \\
$\tilde{\tilde{e}}_{1}$ & $(1.9,1.5,3.5,6,0.5,1)$ & $\tilde{\tilde{d}}_{222}$ & $(56,52,54,56,0.5,1)$ \\
\hline
\end{tabular}

\section{Numerical experiment}

\section{Input data}

In the experiment, assume that there are two vendors $M=2$, two customers $N=2$ and two conveyances $K=2$. Let unit transportation costs, availabilities of product, demands of product, capacities of conveyances, unit transportation time and unit loading and unloading times are type- 2 trapezoidal fuzzy in nature and these are given in Table 1 .

\section{Optimum results}

With the above input data from Table 1, the objective equations together with crisp constraints are separately solved using GRG technique and we have listed the values of $f_{i}^{0}$ and $f_{i}^{1},(i=1,2)$. Then these values are given as follows.

For optimum value $\xi_{1}$ :

$$
f_{1}^{0}=286.9234 \quad f_{1}^{1}=342.6957 \quad f_{2}^{0}=127.3053 \quad f_{2}^{1}=78.14594
$$

So, we formulate the membership functions for $f_{1}$ and $f_{2}$ as follows

$$
\mu_{1}\left(f_{1}(x)\right)=\left\{\begin{array}{cll}
1, & \text { for } & f_{1}(x)<286.9234 \\
\frac{342.6957-f_{1}(x)}{342.6957-286.9234}, & \text { for } & 286.9234 \leq f_{1}(x) \leq 342.6957 \\
0, & \text { for } & f_{1}(x)>342.6957
\end{array}\right.
$$

Table 2 Optimistic value $\left(\xi_{1}\right)$ of $f_{1}$ and $f_{2}$ via GPT

\begin{tabular}{llll}
\hline$\mu_{1}$ & $\mu_{2}$ & $f_{1}$ & $f_{2}$ \\
\hline 0.1 & 0.1 & 337.1185 & 151.4548 \\
& 0.5 & 337.1185 & 151.5363 \\
& 0.9 & 340.49 & 122.5435 \\
0.5 & 0.1 & 325.6547 & 137.1486 \\
& 0.5 & 325.6446 & 137.1561 \\
& 0.9 & 327.1125 & 115.3108 \\
0.9 & 0.1 & 296.1603 & 173.051 \\
& 0.5 & 299.6982 & 150.2512 \\
& 0.9 & 330.7623 & 119.9491 \\
\hline
\end{tabular}


Table 3 Pessimistic value $\left(\xi_{2}\right)$ of $f_{1}$ and $f_{2}$ via GPT

\begin{tabular}{llll}
\hline$\mu_{1}$ & $\mu_{2}$ & $f_{1}$ & $f_{2}$ \\
\hline 0.1 & 0.1 & 338.8941 & 149.8294 \\
& 0.5 & 338.8941 & 149.8435 \\
& 0.9 & 338.8941 & 131.8406 \\
0.5 & 0.1 & 316.2694 & 152.1988 \\
& 0.5 & 320.0879 & 149.1702 \\
& 0.9 & 334.962 & 120.2972 \\
0.9 & 0.1 & 326.0772 & 177.8517 \\
& 0.5 & 306.0398 & 146.1588 \\
& 0.9 & 332.1073 & 121.1518 \\
\hline
\end{tabular}

$$
\mu_{2}\left(f_{2}(x)\right)=\left\{\begin{array}{cll}
1, & \text { for } & f_{2}(x)<78.14594 \\
\frac{127.3053-f_{2}(x)}{127.3053-78.14594}, & \text { for } & 78.14594 \leq f_{2}(x) \leq 127.3053 \\
0, & \text { for } & f_{2}(x)>127.3053
\end{array}\right.
$$

For pessimistic value $\xi_{2}$ :

$$
f_{1}^{0}=286.2006 \quad f_{1}^{1}=344.7489 \quad f_{2}^{0}=123.9177 \quad f_{2}^{1}=76.86776
$$

So we formulate the membership functions for $f_{1}$ and $f_{2}$ as follows

$$
\begin{aligned}
& \mu_{1}\left(f_{1}(x)\right)=\left\{\begin{array}{cll}
1, & \text { for } & f_{1}(x)<286.2006 \\
\frac{344.7489-f_{1}(x)}{344.7489-286.2006}, & \text { for } & 286.2006 \leq f_{1}(x) \leq 344.7489 \\
0, & \text { for } & f_{1}(x)>344.7489
\end{array}\right. \\
& \mu_{2}\left(f_{2}(x)\right)=\left\{\begin{array}{cll}
1, & \text { for } & f_{2}(x)<76.86776 \\
\frac{123.9177-f_{2}(x)}{123.9177-76.86776}, & \text { for } & 76.86776 \leq f_{2}(x) \leq 123.9177 \\
0, & \text { for } & f_{2}(x)>123.9177
\end{array}\right.
\end{aligned}
$$

For $\mathrm{CV}$ value $\xi_{3}$ :

$$
f_{1}^{0}=277.7770 \quad f_{1}^{1}=343.8440 \quad f_{2}^{0}=125.4119 \quad f_{2}^{1}=77.43150
$$

Table $4 \mathrm{CV}$ value $\left(\xi_{3}\right)$ of $f_{1}$ and $f_{2}$ via GPT

\begin{tabular}{llll}
\hline$\mu_{1}$ & $\mu_{2}$ & $f_{1}$ & $f_{2}$ \\
\hline 0.1 & 0.1 & 337.2373 & 163.3666 \\
& 0.5 & 337.2373 & 140.0457 \\
& 0.9 & 340.6428 & 122.9292 \\
0.5 & 0.1 & 310.8105 & 166.2521 \\
& 0.5 & 324.3868 & 136.0942 \\
& 0.9 & 316.5124 & 141.5057 \\
0.9 & 0.1 & 294.8195 & 170.5921 \\
& 0.5 & 297.6135 & 142.1535 \\
& 0.9 & 314.6268 & 134.4193 \\
\hline
\end{tabular}


Table 5 Optimistic value $\left(\xi_{1}\right)$ of $f_{1}$ and $f_{2}$ via WSM

\begin{tabular}{llll}
\hline$w_{1}$ & $w_{2}$ & $f_{1}$ & $f_{2}$ \\
\hline 0 & 1 & 342.6957 & 78.14594 \\
1 & 0 & 286.9234 & 127.3053 \\
0.1 & 0.9 & 342.6957 & 78.14594 \\
0.3 & 0.7 & 342.6957 & 78.14594 \\
0.5 & 0.5 & 288.1229 & 121.9538 \\
0.7 & 0.3 & 287.6876 & 125.3376 \\
0.9 & 0.1 & 287.6876 & 125.3376 \\
\hline
\end{tabular}

So we formulate the membership functions for $f_{1}$ and $f_{2}$ as follows

$$
\begin{aligned}
& \mu_{1}\left(f_{1}(x)\right)=\left\{\begin{array}{cll}
1, & \text { for } & f_{1}(x)<277.7770 \\
\frac{343.8440-f_{1}(x)}{343.8440-277.7770}, & \text { for } & 277.7770 \leq f_{1}(x) \leq 343.8440 \\
0, & \text { for } & f_{1}(x)>343.8440
\end{array}\right. \\
& \mu_{2}\left(f_{2}(x)\right)=\left\{\begin{array}{cll}
1, & \text { for } & f_{2}(x)<77.43150 \\
\frac{125.4119-f_{2}(x)}{125.4119-77.43150}, & \text { for } & 77.43150 \leq f_{2}(x) \leq 125.4119 \\
0, & \text { for } & f_{2}(x)>125.4119
\end{array}\right.
\end{aligned}
$$

Then, we formulate the following model with the help of interactive goal programming technique (for $\mathrm{CV}$ ):

$$
\left\{\begin{array}{l}
\min _{x}\left[d_{1}^{-}+d_{2}^{-}\right] \\
\text {s.t }\left\{\begin{array}{l}
\frac{343.8440-f_{1}(x)}{343.8440-277.7770}+d_{1}^{+}-d_{1}^{-}=\mu_{1} \\
\frac{15.4119-f_{2}(x)}{125.4119-77.43150}+d_{2}^{+}-d_{2}^{-}=\mu_{2} \\
\text { and }(51) \\
d_{i}^{+} d_{i}^{-}=0, d_{i}^{+}, d_{i}^{-} \geq 0, i=1,2 .
\end{array}\right.
\end{array}\right.
$$

The above single objective function has been solved using LINGO-14.0. The optimal results are reported in Tables 2, 3, and 4. Again using weighted sum method, the problem has been solved using LINGO-14.0 and the optimum results has been tabulated in Tables 5, 6, and 7, by which decision maker choose their expected results by choosing different weights.

\section{Discussion}

From experiments, we determined compromise solutions using goal programming technique and weighted sum method for different optimistic levels $\mu_{1}, \mu_{2}$ and different spreads $\theta_{l}, \theta_{r}$. In order to validate the results, we obtained a sensitivity analysis with different spreads $\theta_{l}, \theta_{r}$. The optimum results are given in Table 8 , where it is observed that the transportation times are decreasing as optimistic levels are increased.

Table 6 Pessimistic value $\left(\xi_{2}\right)$ of $f_{1}$ and $f_{2}$ via WSM

\begin{tabular}{llll}
\hline$w_{1}$ & $w_{2}$ & $f_{1}$ & $f_{2}$ \\
\hline 0 & 1 & 344.7489 & 76.86776 \\
1 & 0 & 286.2006 & 123.9177 \\
0.1 & 0.9 & 344.7489 & 76.86776 \\
0.3 & 0.7 & 344.7489 & 76.86776 \\
0.5 & 0.5 & 287.8086 & 119.3824 \\
0.7 & 0.3 & 286.9582 & 123.0566 \\
0.9 & 0.1 & 286.9582 & 123.0566 \\
\hline
\end{tabular}


Table $7 \mathrm{CV}$ value $\left(\xi_{3}\right)$ of $f_{1}$ and $f_{2}$ via WSM

\begin{tabular}{llll}
\hline$w_{1}$ & $w_{2}$ & $f_{1}$ & $f_{2}$ \\
\hline 0 & 1 & 343.844 & 77.4315 \\
1 & 0 & 277.777 & 125.4119 \\
0.1 & 0.9 & 343.844 & 77.4315 \\
0.3 & 0.7 & 311.05 & 96.44564 \\
0.5 & 0.5 & 279.9655 & 120.51 \\
0.7 & 0.3 & 279.298 & 124.0653 \\
0.9 & 0.1 & 277.777 & 125.4119 \\
\hline
\end{tabular}

If the spreads, both left and right, $\theta_{l}, \theta_{r}$ of T2TrFV are increased, then cost and time decreases and if the spreads are considered as zero, then the proposed model has been converted to type- 1 fuzzy model. It shows that the presented algorithm is competent in finding good solutions and the obtained Pareto optimal solutions set is adequate for decision support systems. The optimistic, pessimistic, and CV values of the objective functions $f_{1}$ and $f_{2}$ are shown Figs. 1 and 2, respectively, with the change of $\mu_{1}$, and $\mu_{2}$. It is observed that $\mathrm{CV}$ values are best out of three values, which is expected.

\section{Conclusions}

In our analysis, we have formulated a single layered distribution system in a solid transportation problem (STP) under type 2 trapezoidal fuzzy environment (T2TrF). Here the capacities of supply at origins, demands at destinations and conveyance capacities, transportation costs per unit, unit transportation time and unit loading and unloading time to be T2TrFVs due to the demand of real life situations. Then the STP is reformulated as cost minimization and transportation time minimization problem by the credibility measure and expectation theory via critical value reduction method. The numerical experiments illustrated the operation and efficiency of the suggested approaches. The corresponding crisp model is solved using GRG (Lingo-14.0).

Table 8 Optimized values of objectives with respect to different spreads via GPT

\begin{tabular}{lllll}
\hline Optimistic value $\left(\xi_{1}\right)$ & $\theta_{r}$ & Fuzzy & $f_{1}$ & $f_{2}$ \\
$\theta_{l}$ & 0.5 & $T 2$ & 334.4461 & 118.8946 \\
0.5 & 0.3 & $T 2$ & 334.5625 & 119.4512 \\
0.7 & 0.9 & $T 2$ & 327.1870 & 115.0227 \\
0.9 & 0 & $T 1$ & 316.5965 & 141.4633 \\
0 & & & \\
Pessimistic value $\left(\xi_{2}\right)$ & $\theta_{r}$ & Fuzzy & $f_{1}$ & $f_{2}$ \\
$\theta_{l}$ & 0.5 & T2 & 334.9620 & 120.2972 \\
0.5 & 0.3 & $T 2$ & 333.1573 & 120.4039 \\
0.7 & 0.9 & $T 2$ & 330.3718 & 120.4579 \\
0.9 & 0 & $T 1$ & 316.5965 & 141.4633 \\
0 & & & & \\
CV value $\left(\xi_{3}\right)$ & $\theta_{r}$ & Fuzzy & $f_{1}$ & $f_{2}$ \\
$\theta_{l}$ & 0.5 & $T 2$ & 336.5965 & 141.4633 \\
0.5 & 0.3 & T2 & 326.6765 & 141.4223 \\
0.7 & 0.9 & T2 & 316.5965 & 141.4633 \\
0.9 & 0 & T1 & 316.5965 & 141.4633 \\
0 & & & \\
\hline
\end{tabular}




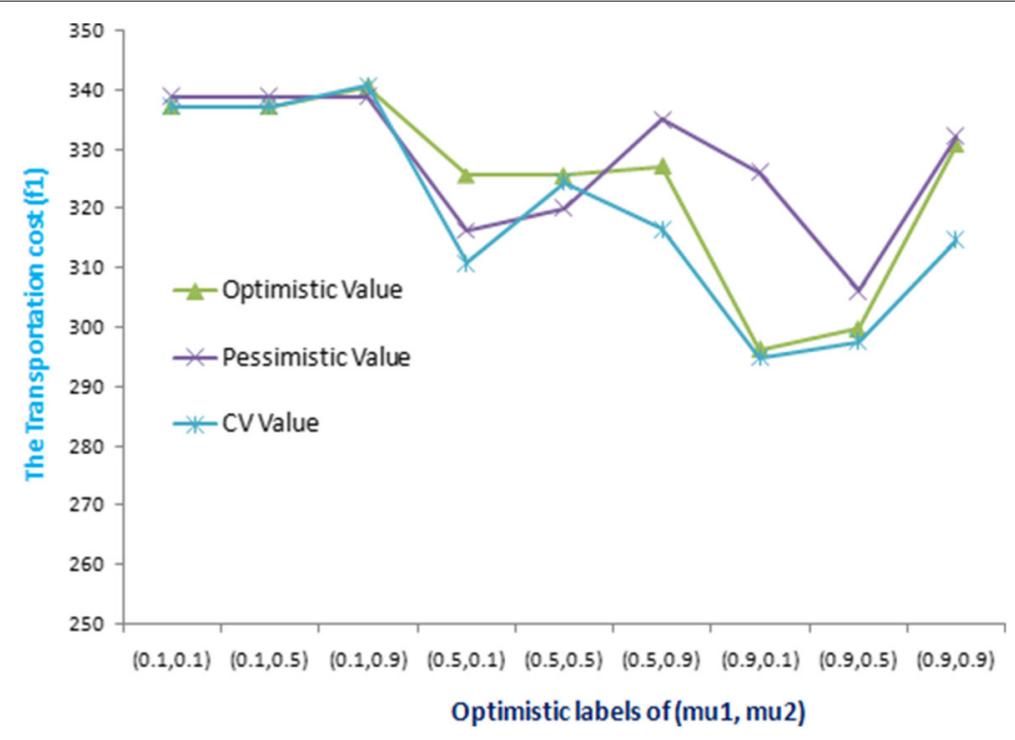

Fig. 1 Different optimistic levels vs transportation cost

The major new features of the paper include the following three aspects:

(i) Expectation formulas of the reductions for type-2 trapezoidal fuzzy variables have been deduced.

(ii) Using the proposed critical value reduction method, a new class of generalized credibility solid transportation problem has been established.

(iii) We have introduced solid transportation problem in T2TrF environments for the first time.

The present work can be continued to multi-item STP and multi-objective STP in two stage distribution system. The presented models can be extended to different types of STPs including transportation time constraints, price discount, breakable/deteriorating items, damageable item, transportation with restriction on transported amount etc.

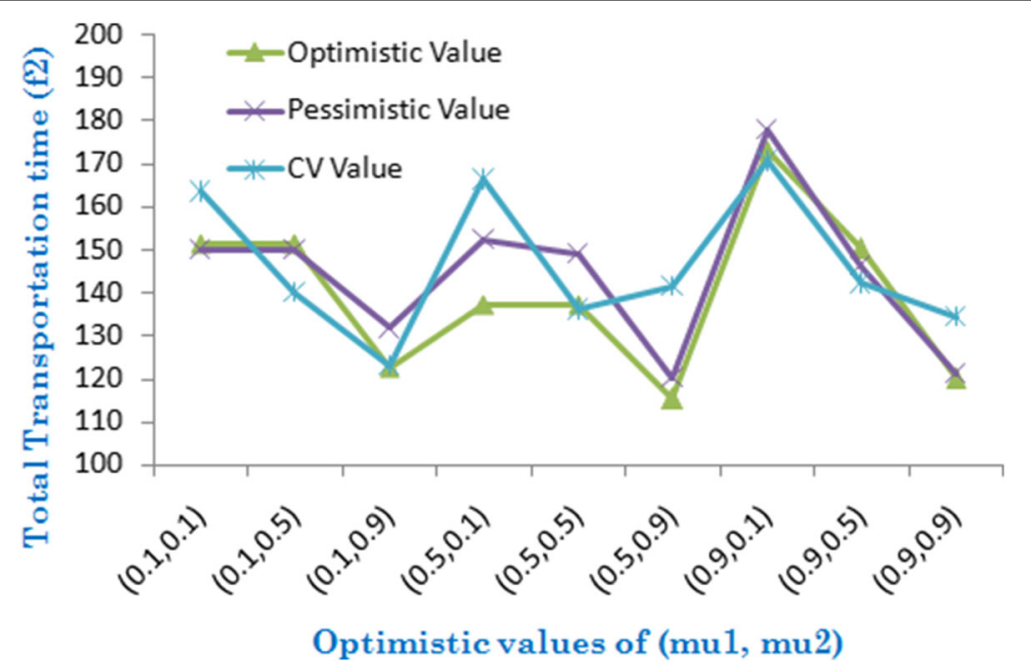

Fig. 2 Different optimistic levels vs transportation time 


\section{Competing interests}

The authors declare that they have no competing interests.

\section{Author details}

${ }^{1}$ Department of BCA, B. P. Poddar Institute of Management and Technology, Saltlake Campus, Kolkata, Pin-700091, West Bengal, India. ${ }^{2}$ Department of Applied Science, Haldia Institute of Technology, Haldia, Purba Midnapur, Pin-721657, West Bengal, India.

Received: 11 November 2016 Accepted: 15 February 2017

Published online: 03 March 2017

\section{References}

1. Zadeh, L.A: The concept of a linguistic variable and its applications in approximate reasoning-I. Inform. Sci. 8(3), 199-249 (1975)

2. Karnik, N.N, Mendel, J.M, Liang, Q.: Type-2 fuzzy logic systems. IEEE Trans. Fuzzy Syst. 7(6), 643-58 (1999)

3. Liang, Q., Mendel, J.M: Interval type-2 fuzzy logic systems: theory and design. IEEE Trans. Fuzzy Syst. 8(5), 535-50 (2000)

4. Karnik, N.N, Mendel, J.M: Operations on type-2 fuzzy sets. Fuzzy Sets Syst. 122(2), 327-48 (2001)

5. Chen, S., Chang, Y.: Fuzzy rule interpolation based on the ratio of fuzziness of interval type-2 fuzzy sets. Expert Syst. Appl. 38(10), 12202-2213 (2011)

6. Abdullah, L., Najib, L.: A new type-2 fuzzy set of linguistic variables for the fuzzy analytic hierarchy process. Expert Syst. Appl. 41(7), 3297-305 (2014)

7. Zadeh, L.A: Fuzzy sets. Inform. Control. 8(3), 338-53 (1965)

8. Karnik, N.N, Mendel, J.M: Centroid of a type-2 fuzzy set. Inform. Sci. 132(1), 195-220 (2001)

9. Coupland, S.: Type-2 Fuzzy sets: geometric defuzzification and type-reduction. Foundations Comput. Intell. 1, 622-29 (2007)

10. Liu, F.: An efficient centroid type-reduction strategy for general type-2 fuzzy logic system. Inform. Sci. 178(9), 2224-236 (2008)

11. Qin, R., Liu, Y., Liu, Z.: Methods of critical value reduction for type-2 fuzzy variables and their applications. J. Comput. Appl. Math. 235(5), 1454-481 (2011)

12. Chen, Y.: The generalized expectations of the reductions for type-2 fuzzy variable. Machine Learning and Cybernetics (ICMLC), 2011 International Conference on. Vol. 1. IEEE, (2011)

13. Haley, K.: The solid transportation problem. Oper. Res. 10,448-63 (1962)

14. Chanas, S., Kolosziejczyj, W., Machaj, A.: A fuzzy approach to the transportation problem. Fuzzy Sets Syst. 13, $211-21$ (1984)

15. Sakawa, M.: Interactive fuzzy goal programming for multiobjective nonlinear programming problems and its applications to water quality management. Control Cybernet. 13(2), 217-28 (1984)

16. Ojha, A., Das, B., Mondal, S., Maiti, M.: A solid transportation problem for an item with fixed charge, vechicle cost and price discounted varying charge using genetic algorithm. Appl. Soft Comput. 10(1), 100-10 (2010)

17. Fegad, M.R, Jadhav, A.V, Minley, A.R: Finding an optimal solution of transportation problem using interval and triangular membership functions. Eur. J. Oper. Res. 60, 415-21 (2011)

18. Molla-Alizadeh-Zavardehi, S., Hajiaghaei-Keshteli, M., Tavakkoli-Moghaddam, R.: Solving a capacitated fixed-charge transportation problem by artificial immune and genetic algorithms with a Prfer number representation. Expert Syst. Appl. 38(8), 10462-0474 (2011)

19. Figueroa-Garca, J.C, Hernndez, G.: A transportation model with interval type-2 fuzzy demands and supplies. International Conference on Intelligent Computing. Springer Berlin Heidelberg (2012)

20. Kaur, A., Kumar, A.: A new approach for solving fuzzy transportation problems using generalized trapezoidal fuzzy numbers. Appl. Soft Comput. 12(3), 1201-213 (2012)

21. Jana, D.K, Maity, K., Roy, T.K: A three-layer supply chain integrated production-inventory model under permissible delay in payments in uncertain environments. J. Uncertainty Anal. Appl. 1(6) (2013)

22. Tao, Z., Xu, J.: A class of rough multiple objective programming and its application to solid transportation problem. Inform. Sci. 188, 215-35 (2012)

23. Kundu, P., Kar, S., Maiti, M.: A fixed charge transportation problem with type-2 fuzzy variables. Inform. Sci. 255 $170-86(2014)$

24. Sadeghi, J., Sadeghi, S., Niaki, S.TA: Optimizing a hybrid vendor-managed inventory and transportation problem with fuzzy demand: an improved particle swarm optimization algorithm. Inform. Sci. 272, 126-44 (2014)

25. Liu, P., Yang, L., Wang, L., Li, S.: A solid transportation problem with type-2 fuzzy variables. Appl. Soft Comput. 24, 543-58 (2014)

26. Yang, L., Liu, P., Li, S., Gao, Y., Ralescu, D.A: Reduction methods of type-2 uncertain variables and their applications to solid transportation problem. Inform. Sci. 291, 204-37 (2015)

27. Jana, D.K, Pramanik, S., Maiti, M.: Mean and CV reduction methods on Gaussian type-2 fuzzy set and its application to a multilevel profit transportation problem in a two-stage supply chain network. Neural Comput. Appl. 1-24 (2016) doi:10.1007/s00521-016-2202-2

28. Mendel, J.M: Advances in type-2 fuzzy sets and systems. Inform. Sci. 177(1), 84-110 (2007)

29. Takc, Z:: Aggregation of fuzzy truth values. Inform. Sci. 271, 1-13 (2014)

30. Jana, D.K, Castillo, O., Pramanik, S., Maiti, M.: Application of interval type-2 fuzzy logic to polypropylene business policy in a petrochemical plant in India. J. Saudi Soc. Agric. Sci (2016). http://dx.doi.org/10.1016/j.jssas.2015.12.004

31. Liu, Q., Liu, Y.: Type-2 fuzzy variables and their arithmetic. Soft Comput. 14(7), 729-47 (2009) 